\title{
SPATIALLY PERIODIC AND APERIODIC MULTI-PULSE PATTERNS IN THE ONE-DIMENSIONAL GIERER-MEINHARDT EQUATION
}

\author{
ARJEN DOELMAN*, TASSO J. KAPER ${ }^{\dagger}$, AND HARMEN VAN DER PLOEG ${ }^{\ddagger}$
}

\begin{abstract}
The generalized Gierer-Meinhardt equation is a paradigm model of two coupled reaction-diffusion equations in the theory of biological pattern formation. The existence of time independent, spatially periodic patterns of this equation in one space dimension is governed by a four-dimensional singularly perturbed ordinary differential equation (ODE). In this paper, we analyze this ODE and use geometric singular perturbation theory to construct countably many different oneparameter families of periodic solutions. These periodic orbits consist of fast segments interspersed with slow segments, such that during the fast segments the concentration of one species exhibits finite sequences of large amplitude pulses that occur in rapid succession and that are of one of two fundamental types while the concentration of the other species is constant. Moreover, during the slow segments, the first species is near equilibrium while the concentration of the second decreases slowly. Most significantly, these fast-slow periodic orbits are shown to exist for all periodic sequences of pulses with two reflection symmetries. Hence, the Gierer-Meinhardt model possesses a rich set of stationary, spatially periodic, multi-pulse patterns. We also treat the case of bounded intervals with Neumann boundary conditions. We show that, given any random sequence of the two pulse types, there exists a stationary, in general aperiodic, multi-pulse pattern with the pulses ordered precisely as in the random sequence on sufficiently long intervals.
\end{abstract}

1. Introduction. In this paper, we study the existence problem for positive, stationary, spatially periodic, multi-pulse solutions $\left(U^{\text {per }}(x), V^{\text {per }}(x)\right)$ of the generalized Gierer-Meinhardt equation

$$
\left\{\begin{array}{l}
U_{t}=U_{x x}-\mu U+U^{\alpha_{1}} V^{\beta_{1}} \\
V_{t}=\varepsilon^{2} V_{x x}-V+U^{\alpha_{2}} V^{\beta_{2}}
\end{array}\right.
$$

for $x \in \mathbf{R}[17,23,24,16,29,30]$ (see also [5] and Remark 1.2). The unboundedness of the domain reflects our choice to study a spatially extended system: the spatial scale of the patterns is assumed to be much smaller than the length scale of the domain. The ratio, $\varepsilon^{2}$, of the diffusion coefficients of the two species, whose concentrations are denoted $U$ and $V$, is assumed to be asymptotically small, i.e., $0<\varepsilon \ll 1$. Throughout this paper, the parameters $\left(\alpha_{1}, \alpha_{2}, \beta_{1}, \beta_{2}\right)$ and $\mu$ are assumed to satisfy

$$
\alpha_{1}>1+\frac{\alpha_{2} \beta_{1}}{\beta_{2}-1}, \alpha_{2}<0, \beta_{1}>1, \beta_{2}>1, \mu>0
$$

(compare to $[17,23,24,16,29,30]$ ). This model can be seen as a generalization of the original model introduced by Gierer and Meinhardt [13] in the context of morphogenesis. The special case $\alpha_{1}=0, \alpha_{2}=-1, \beta_{1}=2, \beta_{2}=2$ in (1.1) corresponds to the original (biological) values of the parameters in [13]. We have used this choice of parameters in the simulations that generated Figures 1.1a and 1.1b.

Multi-pulse solutions of (1.1) will in general have asymptotically large amplitude [5]. Therefore, we introduce the $\mathcal{O}(1)$ functions $\tilde{U}(x, t)$ and $\tilde{V}(x, t)$ and parameters

\footnotetext{
${ }^{*}$ Korteweg-de Vries Instituut, Universiteit van Amsterdam, Plantage Muidergracht 24, 1018 TV Amsterdam, the Netherlands.

$\dagger$ Department of Mathematics, Boston University, 111 Cummington Street, Boston, MA 02215, U.S.A.

${ }^{\ddagger}$ Korteweg-de Vries Instituut, Universiteit van Amsterdam, Plantage Muidergracht 24, 1018 TV Amsterdam, the Netherlands.
} 

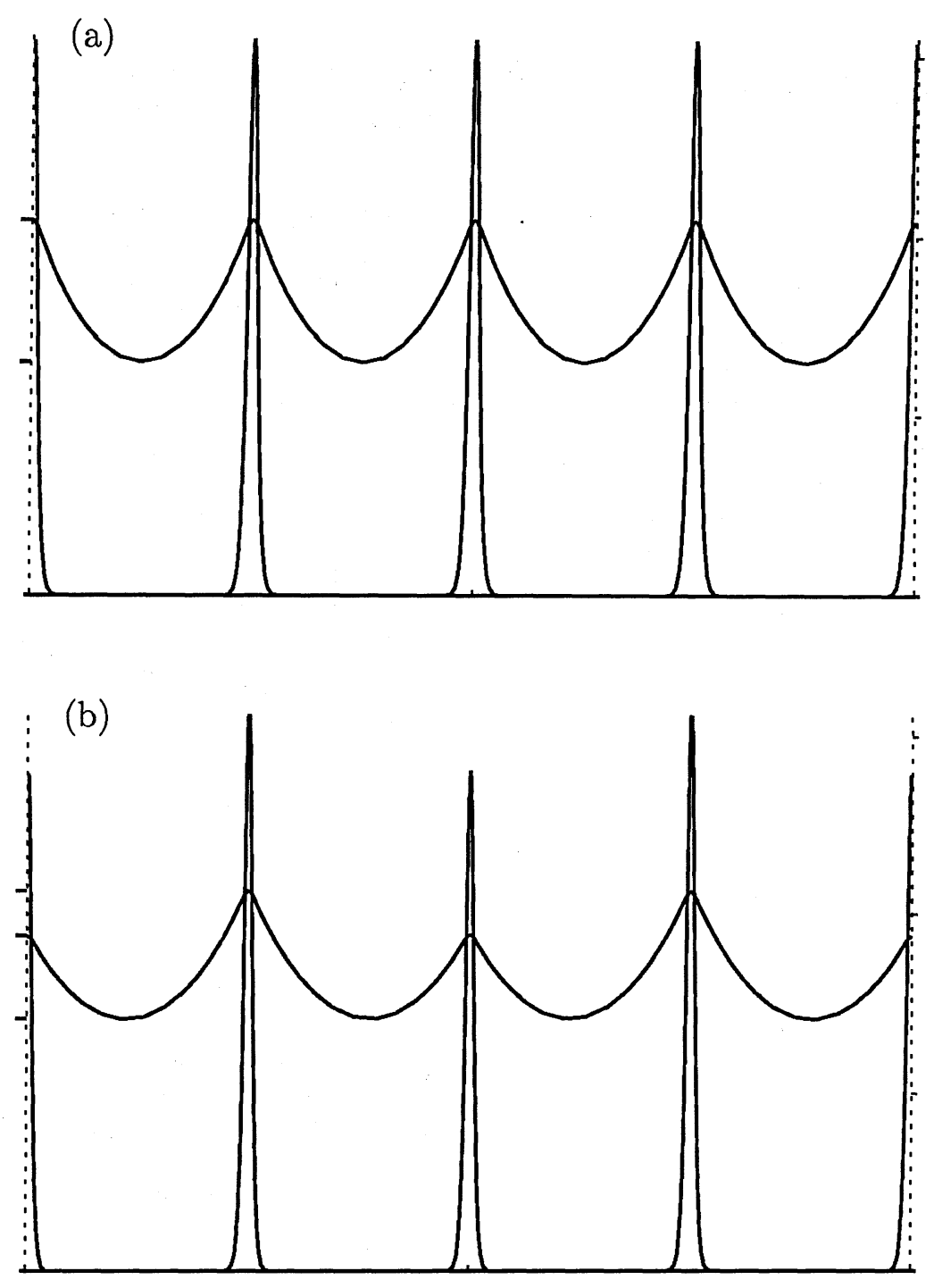

FIG. 1.1. (a) A (finite part of a) fundamental periodic pattern $\left(U_{A_{1}}^{\text {per }}(x), V_{A_{1}}^{\text {per }}(x)\right)$ that is (numerically) stable as solution of the scaled PDE (1.4). (b) A (numerically) stable periodic pattern of 'mixed' type: the $A_{1} B_{1}$-solution ( $U_{A_{1} B_{1}}^{\text {per }}(x), V_{A_{1} B_{1}}^{\text {per }}(x)$ ). Both graphs were obtained by a direct numerical integration of (1.4) with $\alpha_{1}=0, \alpha_{2}=-1, \beta_{1}=\beta_{2}=0$ and $\mu=1$ on a bounded domain of length $L=50$, using the moving-grid code described in [1]. In (a) $\varepsilon^{2}=0.0300$, in (b) $\varepsilon^{2}=0.0206$. Note that both patterns can be extended to solutions on $\mathbf{R}$ by reflection with respect to the boundaries of the domain.

$r, s>0$ by

$$
\tilde{U}(x, t)=\varepsilon^{r} U(x, t), r>0, \quad \tilde{V}(x, t)=\varepsilon^{s} V(x, t), s>0,
$$

and perform a scaling analysis. It is shown in [5] that it is possible to construct stationary, multi-pulse homoclinic solutions of (1.1) for

$$
r=\frac{\beta_{2}-1}{D}>0, s=-\frac{\alpha_{2}}{D}>0, \text { and } D=\left(\alpha_{1}-1\right)\left(\beta_{2}-1\right)-\alpha_{2} \beta_{1}>0
$$


(see [17, 16] for a similar rescaling). Moreover, there generally exists a separation of length scales with the pulses occupying a narrow region and the intervals on either side of the pulse region being long. Hence, it is also natural to rescale the independent variable as $x=\sqrt{\varepsilon} \tilde{x}$ and to consider $\tilde{\varepsilon}=\sqrt{\varepsilon}$. In terms of this new variable, (1.1) may now be written in the 'normal form':

$$
\left\{\begin{array}{rll}
\varepsilon^{2} U_{t} & =U_{x x}-\varepsilon^{2} \mu U & +U^{\alpha_{1}} V^{\beta_{1}} \\
V_{t} & =\varepsilon^{2} V_{x x}-V & +U^{\alpha_{2}} V^{\beta_{2}}
\end{array}\right.
$$

where we have dropped the tildes. Equation (1.4) is the subject of the analysis in this paper, and one directly observes that it has the reversibility symmetry $x \rightarrow-x$, just like (1.1) does, which plays a crucial role in the analysis.

Since $x \in \mathbf{R}$, the existence problem for stationary solutions of (1.4) can be written as a singularly perturbed ordinary differential equation in which $x$ plays the role of 'time':

$$
\left\{\begin{aligned}
u^{\prime} & =p \\
p^{\prime} & =-u^{\alpha_{1}} v^{\beta_{1}}+\varepsilon^{2} \mu u \\
\varepsilon v^{\prime} & =q \\
\varepsilon q^{\prime} & =v-u^{\alpha_{2}} v^{\beta_{2}}
\end{aligned}\right.
$$

where ' denotes the derivative with respect to the slow spatial variable $x$. By introducing the fast variable $\xi=x / \varepsilon$, we can write the slow system (1.5) in the equivalent fast form

$$
\left\{\begin{aligned}
\dot{u} & =\varepsilon p \\
\dot{p} & =\varepsilon\left[-u^{\alpha_{1}} v^{\beta_{1}}+\varepsilon^{2} \mu u\right] \\
\dot{v} & =q \\
\dot{q} & =v-u^{\alpha_{2}} v^{\beta_{2}}
\end{aligned}\right.
$$

where ${ }^{\circ}$ denotes the derivative with respect to $\xi$. Both equations inherit the reversibility symmetry of (1.1) and (1.4):

$$
x, \xi \rightarrow-x,-\xi, \quad p \rightarrow-p, q \rightarrow-q .
$$

The systems $(1.6) /(1.5)$ have one fixed point, $S$, at $(0,0,0,0)$. This fixed point lies on the boundary of the slow manifold $\mathcal{M}$, the central feature of interest in $(1.6) /(1.5)$ :

$$
\mathcal{M}=\{(u, p, v, q): v=q=0, u>0\}
$$

(see Remark 1.4). As may be seen by direct inspection, this manifold is invariant under the flow generated by $(1.6) /(1.5)$ (recall that $\beta_{1}, \beta_{2}>0(1.2)$ ).

We will study the existence of periodic solutions $\gamma^{\text {per }}(\xi)$ of $(1.6)$ by the methods of geometric singular perturbation theory $[10,11]$. We refer to $[18,20]$ for an introduction to the general theory and to [26] for a general account on the geometric theory of periodic orbits in singularly perturbed systems. In this paper we use a combination of geometrical and asymptotical techniques that has been developed to establish the existence of periodic/homoclinic/heteroclinic orbits in singularly perturbed ODEs [6], [15], [27], [26] and in Hamiltonian systems [21], [2]. These methods have also been employed to study the existence of (multi-pulse) traveling waves in various PDEs: the Ginzburg-Landau equation [3], coupled Ginzburg-Landau equations [8], the Gray-Scott equation [7], and several families of singularly perturbed reactiondiffusion equations [5], [14]. We note that this list is far from being exhaustive. 
Usually, the search for traveling 'localized' solutions is one of the first steps in the existence analysis of nontrivial solutions of a parabolic PDE. When the PDE is defined for $x \in \mathbf{R}$, these localized patterns correspond to homoclinic or heteroclinic solutions of an associated ODE reduction $((1.5) /(1.6)$ in the case of stationary solutions of (1.4)). The following result on the existence of singular, stationary, multi-pulse homoclinic solutions of the generalized Gierer-Meinhardt equations was proven in [5]:

TheOREM 1.1: HOMOCLINIC MULTI-PUlSE SOlutions. Let $\left(\alpha_{1}, \alpha_{2}, \beta_{1}, \beta_{2}, \mu\right)$ satisfy (1.2). Then, for any $N \geq 1$ with $N=\mathcal{O}(1)$ and $\varepsilon>0$ small enough, (1.6) possesses an $N$-loop orbit $\gamma_{N}^{\text {hom }}(\xi)$ homoclinic to $S=(0,0,0,0)$. The $u, v$ coordinates of $\gamma_{N}^{\text {hom }}(\xi)$ are non-negative, and $\gamma_{N}^{\text {hom }}(\xi)$ is exponentially close to $\mathcal{M}$ except for a connected interval of $\xi$ values during which it makes $N$ circuits through the fast field, and during which it remains at least $\mathcal{O}(\sqrt{\varepsilon})$ away from $\mathcal{M}$.

A solution $\gamma_{N}^{\text {hom }}(\xi)$ corresponds to a localized pulse solution $\left(U_{N}^{\text {hom }}(x), V_{N}^{\text {hom }}(x)\right)$ of the PDE (1.4). When $N=1, V_{N}^{\text {hom }}(x)$ consists of only one sharp 'pulse' on a narrow spatial region; whereas when $N \geq 2, V_{N}^{\text {hom }}(x)$ is a sequence of $N$ consecutive narrow pulses that are relatively close to each other on a narrow spatial region. In particular, $V_{N}^{\text {hom }}(x)$ decreases to $\mathcal{O}(\sqrt{\varepsilon})$, but not further, in between any two adjacent pulses in this narrow region, while outside of it $V_{N}^{\text {hom }}(x)$ is exponentially small. Furthermore, the $U$-component, $U_{N}^{\text {hom }}(x)$, is constant, at its maximum value, to leading order inside the narrow region, and then it decreases to zero outside of this region on a much longer spatial scale (Remark 1.4). Finally, we note that, of course, the $U$ - and $V$-components of $\left(U_{N}^{\text {hom }}(x), V_{N}^{\text {hom }}(x)\right)$ are, by construction, $\mathcal{O}(1)$ as solutions of the scaled PDE (1.4). However, the $U$ and $V$-components of the solution of the original Gierer-Meinhardt equation (1.1) are asymptotically large with respect to $\varepsilon$. More precisely, they are of $\mathcal{O}\left(1 / \varepsilon^{r}\right)$ and $\mathcal{O}\left(1 / \varepsilon^{s}\right)$, respectively, where $r$ and $s$ are given in (1.3).

The orbits $\gamma_{N}^{\text {hom }}(\xi)$ are all homoclinic to the saddle point $S$ of (1.6). In fact, $\gamma_{N}^{\text {hom }}(\xi) \in W^{s}(S) \cap W^{u}(S)$, where $W^{s}(S)$ and $W^{u}(S)$ are the stable and unstable manifolds of $S$. However, both $W^{s}(S)$ and $W^{u}(S)$ are two-dimensional (see Remark 1.4 below, and [5]), while the phase space of (1.6) is four-dimensional. Thus, one should a priori not expect to be able to establish the existence of the intersections $\gamma_{N}^{\text {hom }}(\xi)$. Nonetheless, it can be shown that $W^{s}(S)$ and $W^{u}(S)$ do intersect (countably many times) by the application of the reversibility or reflection symmetry (1.7) (see section 2 of this paper, as well as [5], for the details). In this paper, the symmetry will be used in a similar way to establish the existence of one-parameter families of symmetric periodic patterns.

The first result of this paper (Theorem 3.1) is a natural extension of Theorem 1.1 to spatially periodic multi-pulse solutions. In particular, we show that, for any $N \geq 1$ of $\mathcal{O}(1)$, there exist two one-parameter families of 'fundamental' periodic solutions, $\gamma_{A_{N}}^{\text {per }}(\xi ; C)$ and $\gamma_{B_{N}}^{\text {per }}(\xi ; C)$ of (1.4). Orbits in different families are distinguished by the type of 'jump' they make from $\mathcal{M}$ to $\mathcal{M}$. During its period, an orbit $\gamma_{A_{N}}^{\text {per }}(\xi)$, respectively $\gamma_{B_{N}}^{\text {per }}(\xi)$, 'takes off' once from $\mathcal{M}$, makes $N$ circuits through the fast field of $A_{N}$-type, respectively $B_{N}$-type, and again 'touches down' on $\mathcal{M}$. The orbits $\gamma_{A_{N}}^{\text {per }}(\xi)$ and $\gamma_{B_{N}}^{\text {per }}(\xi)$ are exponentially close to $\mathcal{M}$ after 'touch down' up until the next 'take off'. The 'jump', 'take off', 'touch down' terminology and the details of 'being exponentially close to $\mathcal{M}$ ' are explained in full detail in sections 2 and 3 of this paper. In section 2 , the take off and touch down curves, $T_{N}^{\text {off }}$ and $T_{N}^{\text {down }}\left(\right.$ with $T_{N}^{\text {off, down }} \subset \mathcal{M}$ ) are also defined and determined. These curves, and especially their intersections with the orbits $\Gamma_{C}$ of the slow flow on $\mathcal{M}$ (see section 2.1), determine the difference between the $A_{N}$-jumps and the $B_{N}$-jumps through the fast field. 
The main difference between the periodic $A_{N^{-}}$and $B_{N^{-}}$-patterns $\left(U_{A_{N}}^{\text {per }}(x), V_{A_{N}}^{\text {per }}(x)\right)$ and $\left(U_{B_{N}}^{\text {per }}(x), V_{B_{N}}^{\text {per }}(x)\right)$ of the PDE (1.4), which are associated to the solutions $\gamma_{A_{N}}^{\text {per }}(\xi)$ and $\gamma_{B_{N}}^{\text {per }}(\xi)$, respectively, of (1.6), is that the amplitudes of the $U$ and $V$ components of the $A_{N}$-type pulses are always larger than the amplitudes of the $U$ and $V$ components of the $B_{N}$-type pulses (see Figure 1.1). In turn, the amplitudes of the $\left(U_{A_{N}}^{\text {per }}(x), V_{A_{N}}^{\text {per }}(x)\right)$ and $\left(U_{B_{N}}^{\text {per }}(x), V_{B_{N}}^{\text {per }}(x)\right)$ patterns are always less than the amplitudes of the corresponding homoclinic pattern $\left(U_{N}^{\text {hom }}(x), V_{N}^{\text {hom }}(x)\right)$. Apart from that, the structure of the $V$-component of an $A_{N^{-}}$or $B_{N}$-pulse is, in essence, the same as that of the homoclinic pulse in $V_{N}^{\text {hom }}(x)$, especially in that $V_{A_{N}}^{\text {per }}(x)$ and $V_{B_{N}}^{\text {per }}(x)$ are exponentially small outside the narrow region in which $\gamma_{A_{N}}^{\text {per }}(\xi)$ and $\gamma_{B_{N}}^{\text {per }^{N}}(\xi)$, respectively, make their circuits through the fast field. The $U$-components $U_{A_{N}}^{\text {per }}(x)$ and $U_{B_{N}}^{\text {per }}(x)$ again vary on a long spatial scale. However, unlike the $U$-component $U_{N}^{\text {hom }}(x)$ of a multi-pulse homoclinic orbit, the $U$-component of all of the periodic patterns constructed in this paper remains bounded away from zero (see Figures 1.1a, b and 2).

Each fundamental periodic orbit $\gamma_{\sigma}^{\text {per }}(\xi)$, where $\sigma$ is any type of the form $\left\{A_{N}, B_{M}\right\}_{N, M \geq 1}$, can be considered as a 'skeleton' for the construction of periodic orbits of 'mixed' type. In Figure $1.1 \mathrm{~b}$, we show a plot of the pattern $\left(U_{A_{1} B_{1}}^{\mathrm{per}}(x), V_{A_{1} B_{1}}^{\mathrm{per}}(x)\right)$ that was obtained as the time-asymptotic state in a simulation of the PDE (1.4). This pattern corresponds to the most simple periodic orbit of mixed type in that the solution $\gamma_{A_{1} B_{1}}^{\text {per }}(\xi)$ of (1.6) is a periodic orbit that makes two different 'jumps' through the fast field, one of $A_{1}$-type and the other of $B_{1}$-type. Our second main result, Theorem 3.3, establishes that, for each $N \geq 1$ and $M \geq 1$, there exists a one-parameter family of periodic orbits $\gamma_{\sigma_{1} \sigma_{2}}^{\text {per }}(\xi)$, with $\sigma_{1}, \sigma_{2} \in\left\{A_{N}, B_{M}\right\}$. These orbits are called 'of $\sigma_{1} \sigma_{2}$-type' (see section 3.2 for the details), since the patterns $\left(U_{\sigma_{1} \sigma_{2}}^{\text {per }}(x), V_{\sigma_{1} \sigma_{2}}^{\text {per }}(x)\right)$ associated to these solutions consist of periodic sequences $\ldots \sigma_{1} \sigma_{2} \sigma_{1} \sigma_{2} \sigma_{1} \sigma_{2} \ldots$ of pulses of $\sigma_{1}$-type and $\sigma_{2}$-type (Figure $1.1 \mathrm{~b}$ ).

It follows from the construction of the 'fundamental' orbits of $\sigma$-type and the 'mixed' orbits of $\sigma_{1} \sigma_{2}$-type that there must be many other families of singular, periodic, multi-pulse solutions of (1.6), or, equivalently, many other types of singular, spatially periodic, multi-pulse patterns in the generalized Gierer-Meinhardt equation (1.1).

Of course, these one-parameter families of periodic solutions will have to obey the reversibility (or reflection) symmetry (1.7). Hence, an element of such a family of periodic solutions, $\gamma^{\text {per }}(\xi ; C)$, must satisfy (after translation) $\gamma^{\text {per }}(0 ; C) \in\{p=q=0\}$ and $\gamma^{\text {per }}\left(\frac{T}{2} ; C\right) \in\{p=q=0\}$ where $T=T(C)$ is the period, or wave length, of $\gamma^{\text {per }}(\xi ; C)$. Thus, $\gamma^{\text {per }}(\xi ; C)$ has two internal symmetries, $\gamma^{\text {per }}(-\xi ; C)=\gamma^{\text {per }}(\xi ; C)$ and $\gamma^{\text {per }}\left(\frac{T}{2}-\xi ; C\right)=\gamma^{\text {per }}\left(\frac{T}{2}+\xi ; C\right)$ (we refer to section 3 for the details). It follows that one can distinguish between three possible types of periodic solutions $\gamma^{\text {per }}(\xi ; C)$. An orbit $\gamma^{\text {per }}(\xi ; C)$ might have both $\gamma^{\text {per }}(0 ; C)$ and $\gamma^{\text {per }}\left(\frac{T}{2} ; C\right)$ exponentially close to $\mathcal{M}$, or it might have $\gamma^{\text {per }}(0 ; C)$ exponentially close to $\mathcal{M}$ and $\gamma^{\text {per }}\left(\frac{T}{2} ; C\right)$ in the fast field (note that one can always translate, so that one can assume that $\gamma^{\text {per }}(0 ; C$ ) is close to $\mathcal{M})$; finally, $\gamma^{\text {per }}(\xi ; C)$ might have both $\gamma^{\text {per }}(0 ; C)$ and $\gamma^{\text {per }}\left(\frac{T}{2} ; C\right)$ in the fast field, i.e. each halfway along a fast $\sigma$-jump or $\tilde{\sigma}$-jump from $\mathcal{M}$ to $\mathcal{M}$.

In Theorem 3.4 we give a complete characterization of all possible one-parameter families of symmetric periodic solutions. In particular, let $\tau$ be a 'random' sequence of $\sigma_{j}$ 's, $j=1,2, \ldots, J$, where $\sigma_{j}$ represents a jump through the fast field of $\sigma_{j}$-type, i.e. $\tau=\sigma_{1} \sigma_{2} \ldots \sigma_{J}$ with $\sigma_{j} \in\left\{A_{N}, B_{M}\right\}_{N, M \geq 1}$, and let $\tau^{-1}$ be defined by $\tau^{-1}=$ $\sigma_{J} \sigma_{J-1} \ldots \sigma_{1}$. It is shown in Theorem 3.4 that one can construct three different one- 


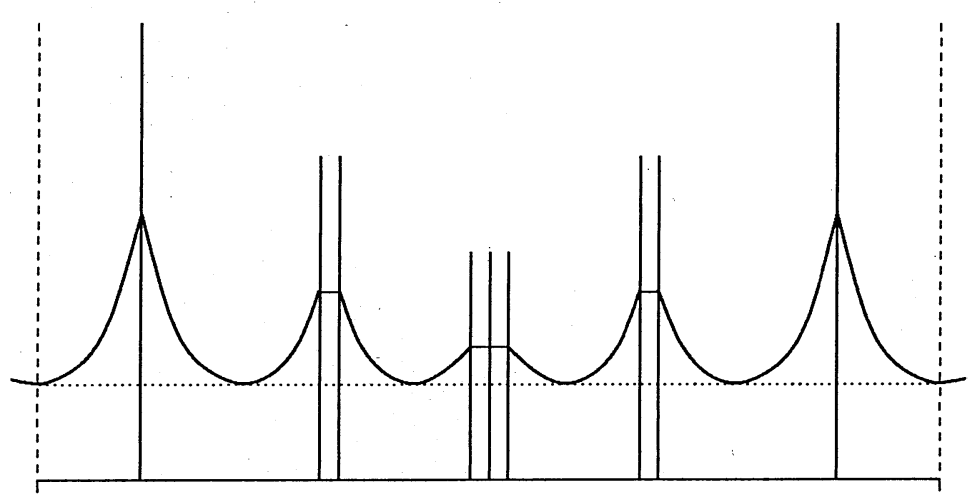

FIG. 1.2. A schematic illustration of a finite part of the pattern $\left(U_{A_{1} A_{2} A_{3} A_{2} A_{1}}^{\mathrm{per}}(x), V_{A_{1} A_{2} A_{3} A_{2} A_{1}}^{\mathrm{per}}(x)\right)$ associated to an $A_{1} A_{2} A_{3} A_{2} A_{1}$-orbit of $\tau \sigma \tau^{-1}$-type (with $\tau=A_{1} A_{2}, \sigma=A_{3}$ ). The 'full' pattern can again be obtained by reflection with respect to the boundaries of the domain. The singular $V$-pulses are represented by vertical line segments. The $U$-components are, to leading order, constant during the fast excursions of $\gamma_{A_{1} A_{2} A_{3} A_{2} A_{1}}^{\text {per }}(\xi)$. Note that the values of $U$ are identical at the minima of $U_{A_{1} A_{2} A_{3} A_{2} A_{1}}^{\text {per }}(x)$ (up to exponentially small corrections, see section 3).

parameter families of singular symmetric periodic orbits for any $\tau$ : one of $\tau \tau^{-1}$-type, one of $\tau \sigma \tau^{-1}$-type and one of $\sigma \tau \tilde{\sigma} \tau^{-1}$-type, where $\sigma$ and $\tilde{\sigma}$ are arbitrary.

The orbits of $\tau \tau^{-1}$-type correspond to solutions $\gamma_{\tau \tau^{-1}}^{\mathrm{per}}(\xi ; C)$ of $(1.6)$ that have both the points $\gamma_{\tau \tau^{-1}}^{\text {per }}(0 ; C)$ and $\gamma_{\tau^{-1}}^{\text {per }}\left(\frac{T}{2} ; C\right)$ exponentially close to $\mathcal{M}$. The associated pattern $\left(U_{\tau \tau^{-1}}^{\text {per }}(x), V_{\tau \tau^{-1}}^{\text {per }}(x)\right)$ in the PDE $(1.1) /(1.4)$ exhibits a periodic sequence of singular pulses of $\sigma_{j}$-type of the form

$$
\ldots \sigma_{1} \sigma_{2} \ldots \sigma_{J} \sigma_{J} \sigma_{J-1} \ldots \sigma_{1} \sigma_{1} \sigma_{2} \ldots \sigma_{J} \sigma_{J} \sigma_{J-1} \ldots \sigma_{1} \ldots
$$

where $\sigma_{j}$ can represent any possible jump through the fast field of $A_{N^{-}}$or $B_{M}$-type. The $\tau \sigma \tau^{-1}$-orbits correspond to solutions $\gamma_{\tau \sigma \tau^{-1}}^{\text {per }}(\xi ; C)$ that have $\gamma_{\tau \sigma \tau^{-1}}^{\text {per }}(0 ; C)$ exponentially close to $\mathcal{M}$ and $\gamma_{\tau \sigma \tau^{-1}}^{\text {per }}\left(\frac{T}{2} ; C\right)$ in the fast field, halfway along a jump of $\sigma$-type. In Figure 1.2, a sketch is given of the pulse pattern ... $A_{1} A_{2} A_{3} A_{2} A_{1} A_{1} A_{2} A_{3} A_{2} A_{1} \ldots$ generated by $\left(U_{A_{1} A_{2} A_{3} A_{2} A_{1}}^{\mathrm{per}}(x), V_{A_{1} A_{2} A_{3} A_{2} A_{1}}^{\mathrm{per}}(x)\right)$, the solution of (1.4) associated to the $\tau \sigma \tau^{-1}$-orbit $\gamma_{A_{1} A_{2} A_{3} A_{2} A_{1}}^{\text {per }}(\xi ; C)$ with $\tau=A_{1} A_{2}, \sigma=A_{3}$. Finally, the $\sigma \tau \tilde{\sigma} \tau^{-1}$-orbits correspond to orbits $\gamma_{\sigma \tau \tilde{\sigma} \tau^{-1}}^{\text {per }}(\xi ; C)$ that have their intersections with $\{p=q=0\}$ halfway along their jumps of $\sigma$ - and $\tilde{\sigma}$-type. The corresponding patterns $\left(U_{\sigma \tau \tilde{\sigma} \tau^{-1}}^{\mathrm{per}}(x), V_{\sigma \tau \tilde{\sigma} \tau^{-1}}^{\mathrm{per}}(x)\right)$ are generated by the following periodic sequences:

$$
\ldots \sigma \sigma_{1} \sigma_{2} \ldots \sigma_{J} \tilde{\sigma} \sigma_{J} \sigma_{J-1} \ldots \sigma_{1} \sigma \sigma_{1} \sigma_{2} \ldots \sigma_{J} \tilde{\sigma} \sigma_{J} \sigma_{J-1} \ldots \sigma_{1} \sigma \sigma_{1} \sigma_{2} \ldots
$$

with $\sigma, \tilde{\sigma}, \sigma_{j} \in\left\{A_{N}, B_{M}\right\}_{N, M \geq 1}$ for $j=1,2, \ldots, J$.

Therefore, we may conclude by Theorem 3.4 that the generalized GiererMeinhardt equation (1.1) has an extremely rich set of distinct periodic solutions/patterns.

Finally, we extend the results obtained for periodic intervals to the case of the Gierer-Meinhardt equation defined on a bounded interval with homogeneous Neumann boundary conditions. We show that this leads to a very natural distinction between the three types of periodic orbits described by Theorem 3.4. Specifically, we reformulate Theorem 3.4 into a new result, Corollary 4.1 , by restricting $\xi$ to a bounded 
interval. In essence, Corollary 4.1 states that any 'random', and thus in general aperiodic, multi-pulse pattern of $\tilde{J}$ pulses of $\sigma_{j}$-type, with $\sigma_{j} \in\left\{A_{N}, B_{M}\right\}_{N, M \geq 1}$, exists as solution of (1.4) (and hence also of (1.1)) on a bounded interval of sufficient length and with homogeneous Neumann boundary conditions. Moreover, this corollary clearly brings out why such 'random' patterns exist.

The paper concludes with a brief discussion section, in which we comment on the possible stability of the spatially periodic patterns and on some related issues.

REMARK 1.2. It is shown in [5] that the generalized Gierer-Meinhardt equation can be derived, by a scaling analysis, from a large class of singularly perturbed reaction-diffusion equations as the leading order part of a 'normal form'. This class is given by

$$
\left\{\begin{array}{l}
U_{t}=d_{U} U_{x x}+a_{11} U+a_{12} V+H_{1}(U, V) \\
V_{t}=d_{V} V_{x x}+a_{21} U+a_{22} V+H_{2}(U, V)
\end{array}\right.
$$

where $0<d_{V} \ll d_{U}, a_{i j}$ is such that the trivial pattern $(U(x, t), V(x, t)) \equiv(0,0)$ of the linear equation (i.e. $\left.H_{i}(U, V) \equiv 0\right)$ is asymptotically stable, and $H_{i}(U, V)$ is such that certain growth conditions are satisfied (see [5] for the details). As a consequence, all results on the existence of singular, spatially periodic, multi-pulse patterns obtained in this paper for the generalized Gierer-Meinhardt equation also hold for this general class of reaction-diffusion equations.

REMARK 1.3. The (natural) choice to study positive solutions (i.e. $U(x, t), V(x, t)>0)$ does not have an essential influence on the analysis. We refer to [5] for more details on this issue.

REMARK 1.4. The fact that the fixed point $S$ lies on the boundary of $\mathcal{M}$ where $u=0$ introduces a technical difficulty for the application of the Fenichel geometric singular perturbation theory, since the theory applies for $u>0$. Moreover, since $\alpha_{2}<0$ (1.2), there is a singularity in the vector field at $u=0$ that, a priori, could prevent the existence of orbits homoclinic to $S$. Both these issues are treated and resolved in [5]. Since the periodic solutions studied in this paper remain bounded away from the hyperplane $\{u=0\}$, we do not have to consider the singular behavior at $u=0$ in this paper.

2. Geometric preliminaries. In this section, we present the background material on which the construction of the periodic patterns is based. This approach was in essence already developed in [7] for the Gray-Scott model and was generalized in [5] to a large class of singularly perturbed reaction-diffusion system, including the generalized Gierer-Meinhardt equation. Both [7] and [5] are mostly restricted to homoclinic (i.e. non-periodic) patterns.

2.1. The reduced problems and persistence. The fast reduced limit $\varepsilon \downarrow 0$ of (1.6) is given by

$$
\ddot{v}=v-u^{\alpha_{2}} v^{\beta_{2}}
$$

with $u \equiv u^{0}$ and $p \equiv p^{0}$, where $u^{0}, p^{0} \in \mathbf{R}$ are constants. System (2.1) is integrable and has, for $u^{0}>0$, a saddle fixed point at $(v=0, \dot{v}=0)$ that has an orbit $\left(v^{\text {hom }}(\xi), q^{\text {hom }}(\xi)=\dot{v}^{\text {hom }}(\xi)\right)$ homoclinic to it. Note that one has to assume that $\beta_{2}>1$, which is one of the conditions explicitly contained in the hypotheses (1.2).

The slow manifold $\mathcal{M}$ defined in (1.8) is simply the union of the saddle points $(0,0)$ over all $u^{0}>0$ and all $p^{0} \in \mathbf{R}$. The slow manifold is normally hyperbolic relative 
to (1.6) with $\varepsilon=0$ for all $v$. Specifically, with $\varepsilon=0, \mathcal{M}$ has three-dimensional stable and unstable manifolds that are the unions of the two-parameter $\left(u^{0}, p^{0}\right)$ families of one-dimensional stable and unstable manifolds, respectively, of the saddle points $(v, q)=(0,0)$ in $(2.1)$.

The Fenichel persistence theory (see $[10,11,18,20]$ ) implies that system (1.6) with $0<\varepsilon \ll 1$ has a locally invariant, slow manifold, under the condition that the vector field is at least $C^{1}$. Hence, we have to impose (1.2). Here, we know even more already, since the manifold $\mathcal{M}$ is also invariant in the full system (1.6) with $\varepsilon \neq 0$. In addition, the Fenichel theory states that, in the system (1.6) with $0<\varepsilon \ll 1, \mathcal{M}$ has three-dimensional stable and unstable manifolds, which we denote $W^{s}(\mathcal{M})$ and $W^{u}(\mathcal{M})$, and that these manifolds are $\mathcal{O}(\varepsilon)$ close to their $\varepsilon=0$ counterparts.

Generally, the slow reduced problem can be obtained by taking the limit $\varepsilon \rightarrow 0$ in the slow system (1.5). This reduced system governs the (slow) flow on $\mathcal{M}$. However, taking $\varepsilon \rightarrow 0$ in (1.5) yields $u^{\prime \prime} \equiv 0$, so that we conclude that the flow must be super slow on $\mathcal{M}$ (see also [7]). The flow on $\mathcal{M}$ is obtained by setting $v, q=0$ in (1.5):

$$
u^{\prime \prime}=\varepsilon^{2} \mu u
$$

thus $d / d x=\mathcal{O}(\varepsilon)$, where $x$ is the slow variable.

The slow reduced problem (2.2) is linear. On $\mathcal{M}$, there are one-dimensional stable and unstable manifolds (restricted to $\mathcal{M}$ ) that are asymptotic to the saddle $S=(0,0,0,0)$ on the boundary of $\mathcal{M}$ (see Remark 1.4$)$ :

$$
\ell^{u, s}: p= \pm \varepsilon \sqrt{\mu} u \text {. }
$$

The manifolds $\ell^{u, s}$ are especially important for the construction of homoclinic (multipulse) solutions.

Periodic solutions are associated to a family of hyperbolic solutions of (2.2) that lie to the right of $\ell^{u}$ and $\ell^{s}$ in the $u>0$ half-plane. We define the one-parameter family of orbits

$$
\Gamma_{C}:=\left\{p^{2}=\varepsilon^{2}\left(\mu u^{2}-C\right)\right\} \text { for } C \in \mathbf{R} .
$$

Note that $\Gamma_{C}$ corresponds to $\ell^{s} \cup \ell^{u}$ for $C=0$, and that $u$ changes sign on $\Gamma_{C}$ for $C<0$. Since we focus on positive solutions in this paper, we only consider $\Gamma_{C}$ with $C \geq 0$ (see Figure 2.1 and section 2.4). Solutions on these orbits can be expressed explicitly as

$$
u\left(x ; C ; x_{0}\right)=u^{\min }(C) \cosh \left(\varepsilon \sqrt{\mu}\left(x-x_{0}\right)\right)=u^{\min }(C) \cosh \left(\varepsilon^{2} \sqrt{\mu}\left(\xi-\xi_{0}\right)\right),
$$

so that $u\left(x_{0} ; C ; x_{0}\right)=u^{\min }(C)$ and

$$
u\left(x ; C ; x_{0}\right) \geq u^{\min }(C) \stackrel{\text { def }}{=} \sqrt{\frac{C}{\mu}}>0 .
$$

2.2. Periodic solutions and orbits homoclinic to $\mathcal{M}$. Periodic solutions of (1.6) cannot be homoclinic to $\mathcal{M}$. However, we now show that any periodic solution of (1.6) must have a singular structure, in the sense that it must consist of 'slow' parts close to $\mathcal{M}$ and 'fast' parts that are excursions away from $\mathcal{M}$ and back to it.

The argument is based on a Poincaré map approach. A periodic solution $\gamma^{\mathrm{per}}(\xi)$ of $(1.6)$, with $\gamma^{\text {per }}(\xi)=\left(u^{\text {per }}(\xi), p^{\text {per }}(\xi), v^{\text {per }}(\xi), q^{\text {per }}(\xi)\right)$, and with $\mathcal{O}(1)$ period $T$, is 
a closed curve in the phase space, so that

$$
\Delta p\left(\gamma^{\mathrm{per}}\right)=\int_{-T / 2}^{T / 2} \dot{p}\left(\gamma^{\mathrm{per}}(\xi)\right) d \xi=0,
$$

by construction. However, it follows from (1.6) that

$$
\Delta p\left(\gamma^{\text {per }}\right)=-\varepsilon\left(u^{0}\right)^{\alpha_{1}} \int_{-T / 2}^{T / 2}\left(v^{0}\left(\xi ; u^{0}\right)\right)^{\beta_{1}} d \xi+\mathcal{O}\left(\varepsilon^{2}\right),
$$

where $u^{0}>0$ is constant. Hence, such a periodic solution satisfies $\left|u^{\text {per }}(\xi)-u^{0}\right|=\mathcal{O}(\varepsilon)$ for all $\xi$ and the $v$-component, $v^{0}\left(\xi ; u^{0}\right)>0$, is a periodic solution of $(2.1)$ that satisfies $\left|v^{\text {per }}(\xi)-v^{0}\left(\xi ; u^{0}\right)\right|=\mathcal{O}(\varepsilon)$ for $\xi \in(-T / 2, T / 2)$. Therefore, $\Delta p\left(\gamma^{\text {per }}\right)$ cannot vanish for $\varepsilon \ll 1$, since the coefficient on the $\mathcal{O}(\varepsilon)$ term in the formula for $\Delta p$ must be strictly negative. In turn, this implies that $\Delta p\left(\gamma^{\text {per }}\right)$ cannot vanish for $\mu \ll 1 / \varepsilon^{4}$. See Remark 2.1 .

Since we consider here $0<\varepsilon \ll 1$ and $\mu=\mathcal{O}(1)$, we conclude that periodic solutions cannot be close to a periodic solution of the fast reduced problem and cannot have an $\mathcal{O}(1)$ period $T$. Only solutions that have $v^{\text {per }}=0$ to leading order for most of the circuit might be able to satisfy the condition $\Delta p=0$. Such solutions thus have 'slow' components that are close to $\mathcal{M}$ (where $v=0$ ) and 'fast' components that are close to the homoclinic solutions $v^{\text {hom }}\left(\xi ; u^{0}\right)$ of the fast reduced problem.

A 'fast' excursion away from, and back to, $\mathcal{M}$ gives a negative contribution to $\Delta p$ strictly of $\mathcal{O}(\varepsilon)$. This contribution must be 'balanced' by the component $+\varepsilon^{3} \mu u$ (2.1) of $\dot{p}$ that is the leading order contribution of $\Delta p$ for $v=0$. It follows that a potential periodic solution must remain close to $\mathcal{M}$ for $\mathcal{O}\left(1 / \varepsilon^{2}\right)$ 'time' (here 'time' = the fast spatial variable $\xi$ ), so that the $\mathcal{O}\left(\varepsilon^{3}\right)$ component can have a net effect also strictly of $\mathcal{O}(\varepsilon)$. Note that this argument also shows that a spatially periodic solution must have a period of $\mathcal{O}(1 / \varepsilon)$ in the (slow) spatial variable $x$ of the (scaled) PDE in normal form (1.4).

The slow manifold $\mathcal{M}$ is normally hyperbolic, hence, a solution can remain close to $\mathcal{M}$ for $\mathcal{O}\left(1 / \varepsilon^{2}\right)$ time (as measured in $\xi$ ) only if it is exponentially close to $\mathcal{M}$ during its 'passage' near $\mathcal{M}$. Therefore, each segment of a periodic solution must be exponentially close to either $\mathcal{M}$, or, during a circuit through the fast field, to $W^{s}(\mathcal{M}) \cap W^{u}(\mathcal{M})([19,27,26]$ and section 3$)$. This implies that any periodic solution to (2.1) must be exponentially close to a solution homoclinic to $\mathcal{M}$. As a consequence, it is necessary to first develop a theory by which solutions homoclinic to $\mathcal{M}$ can be constructed. The results reviewed below were first reported in [5].

One-circuit orbits homoclinic to $\mathcal{M}$ in the full system (1.6) with $0<\varepsilon \ll 1$ will lie in the transverse intersection of $W^{u}(\mathcal{M})$ and $W^{s}(\mathcal{M})$, and their excursions in the fast field will lie close to a homoclinic orbit of (2.1) for some particular value of $u^{0}$.

In order to detect these solutions, we use a Melnikov method for slowly varying systems ([25, 7, 5]) System (2.1) has a conserved quantity, or energy, given by:

$$
K(\xi)=\frac{1}{2} q^{2}-\frac{1}{2} v^{2}+\frac{1}{\beta_{2}+1} u^{\alpha_{2}} v^{\beta_{2}+1} .
$$

By construction, $\left.K\right|_{\mathcal{M}}=0$, and $K<0$ for orbits inside the homoclinic orbit $\left(v^{\text {hom }}\left(\xi ; u^{0}\right), q^{\text {hom }}\left(\xi ; u^{0}\right)\right)$ of $(2.1)$, while $K>0$ for orbits outside. In addition, a direct calculation yields:

$$
\dot{K}=\varepsilon \frac{\alpha_{2}}{\beta_{2}+1} u^{\alpha_{2}-1} p v^{\beta_{2}+1}
$$


i.e., $\dot{K}=\mathcal{O}(\varepsilon)$. Since $\left.K\right|_{\mathcal{M}} \equiv 0$, any orbit $\gamma(\xi)=(u(\xi), p(\xi), v(\xi), q(\xi))$ of $(1.6)$ that is homoclinic to $\mathcal{M}$ must satisfy the condition

$$
\Delta K\left(u^{0}, p^{0}\right)=\int_{-\infty}^{\infty} \dot{K}(\gamma(\xi)) d \xi=0
$$

Here, without loss of generality, we assume that the orbits $\gamma(\xi)$ homoclinic to $\mathcal{M}$, if they exist, are parameterized such that $\gamma(0)=\left(u^{0}, p^{0}, v^{0}, 0\right)$. Therefore, $(2.8)$ implies:

$$
\int_{-\infty}^{\infty} u^{\alpha_{2}-1} p v^{\beta_{2}+1} d \xi=0 .
$$

The condition (2.10) is exact in the sense that we did not introduce any approximations so far. Moreover, as we shall show, if the zero of $\Delta K$ is a simple one, then the homoclinic orbit $\gamma(\xi)$ lies in the transverse intersection of $W^{s}(\mathcal{M})$ and $W^{u}(\mathcal{M})$.

Now, $W^{s}(\mathcal{M})$ and $W^{u}(\mathcal{M})$ are three-dimensional manifolds. Thus, in the fourdimensional phase space of $(1.6)$, one expects that $W^{s}(\mathcal{M}) \cap W^{u}(\mathcal{M})$ is a twodimensional manifold, or, equivalently, that there is a one-parameter family of orbits $\gamma$ that are homoclinic to $\mathcal{M}$. The analysis carried out in the remainder of this subsection reveals that this is indeed the case as long as (1.2) holds.

Since $W^{s}(\mathcal{M})$ and $W^{u}(\mathcal{M})$ are $\mathcal{O}(\varepsilon)$ close to the $\left(u^{0}, p^{0}\right)$-family of homoclinic orbits to (2.1), both $W^{s}(\mathcal{M})$ and $W^{u}(\mathcal{M})$ intersect the three-dimensional hyperplane $\{q=0\}$ transversely in two-dimensional manifolds, defined as $\mathcal{I}_{-1}(\mathcal{M})$ and $\mathcal{I}_{+1}(\mathcal{M})$, respectively. These manifolds can be parameterized by $\left(u^{0}, p^{0}\right)$ :

$$
\mathcal{I}_{ \pm 1}(\mathcal{M})=\left\{\left(u^{0}, p^{0}, v_{ \pm 1}^{0}\left(u^{0}, p^{0}\right), 0\right), u^{0}>0\right\} \subset\{q=0\}
$$

Thus, for every $u^{0}>0$ and $p^{0} \in \mathbf{R}$, there exists a $v_{-1}^{0}$ such that the solution $\gamma(\xi)$ of (1.6) with $\gamma(0)=\left(u^{0}, p^{0}, v_{-1}^{0}, 0\right)$ satisfies $\lim _{\xi \rightarrow \infty} \gamma(\xi) \in \mathcal{M}$. Similarly, there exists a $v_{+1}^{0}$ such that the solution $\gamma(\xi)$ of $(1.6)$ with $\gamma(0)=\left(u^{0}, p^{0}, v_{+1}^{0}, 0\right)$ satisfies $\lim _{\xi \rightarrow-\infty} \gamma(\xi) \in \mathcal{M}$ (where the superscripts are indices, not powers). Note that the use of the above limits constitutes a slight abuse of notation. The slow manifold $\mathcal{M}$ has a boundary $\partial \mathcal{M}=\{(u, p, v, q): u=v=q=0\}$ (1.8) and the vector field (1.6) can be singular when $u \rightarrow 0$. However, in this paper we are only interested in orbits that have positive $u$-coordinates for all $\xi$, see also section 2.4 (and Remarks 1.3, 1.4).

Having established that the sets $\mathcal{I}_{-1}(\mathcal{M})$ and $\mathcal{I}_{+1}(\mathcal{M})$ are nonempty, we now show they intersect in the hyperplane $\{p=0\}$. We use the homoclinic orbit $\left(v^{\text {hom }}(\xi), q^{\text {hom }}(\xi)\right)$ of $(2.1)$ to approximate the solutions on $W^{u}(\mathcal{M})$ and $W^{s}(\mathcal{M})$. The exact condition (2.10) now implies that, to leading order, one-circuit homoclinic solutions must satisfy:

$$
\left(u^{0}\right)^{\alpha_{2}-1} p^{0} \int_{-\infty}^{\infty}\left(v^{\text {hom }}(\xi)\right)^{\beta_{2}+1} d \xi+\mathcal{O}(\varepsilon)=0 .
$$

The improper integral exists because $v^{\text {hom }}$ converges exponentially to zero as $\xi \rightarrow \pm \infty$, and hence so does the entire integrand. Then, since the integrand in (2.12) is positive and since $u^{0}$ is assumed to be positive, we see that it is only possible to satisfy (2.12) if $p^{0}$ is $\mathcal{O}(\varepsilon)$. In addition, we conclude that

$$
\Delta K\left(u^{0}, p^{0}\right)=\mathcal{O}\left(\varepsilon^{2}\right) \text { for } p^{0}=\mathcal{O}(\varepsilon)
$$

Finally, for the one circuit homoclinic orbits to $\mathcal{M}$, we now show that not only is it necessary that $p^{0}=\mathcal{O}(\varepsilon)$, but it is in fact the case that $p^{0} \equiv 0$. We go back to the 
exact condition (2.10). For any solution $\gamma(\xi)=(u(\xi), p(\xi), v(\xi), q(\xi)) \in W^{u}(\mathcal{M})$ with $\gamma(0)=\left(u^{0}, 0, v_{+1}^{0}\left(u^{0}, 0\right), 0\right) \in \mathcal{I}_{+1}(\mathcal{M})$, the reversibility symmetry $(1.7)$ implies that

$$
u(-\xi)=u(\xi), \quad p(-\xi)=-p(\xi), \quad v(-\xi)=v(\xi), \quad \text { and } \quad q(-\xi)=-q(\xi)
$$

and, hence also, $v_{+1}^{0}=v_{-1}^{0}$. Therefore, along $\gamma(\xi)$, the integrand in (2.10) is an odd function of $\xi$, and the integral vanishes identically. This, in turn, implies that $W^{u}(\mathcal{M}) \cap W^{s}(\mathcal{M})$ precisely in the orbit $\gamma(\xi)$ and that the set $\mathcal{I}_{+1}(\mathcal{M}) \cap \mathcal{I}_{-1}(\mathcal{M}) \subset$ $\{p=0\}$.

REMARK 2.1. In [22] the transition from singular periodic orbits to regular periodic orbits (by varying the parameters), and the ultimate 'fate' of the family of periodic orbits in a Turing bifurcation, has been analyzed in full detail for the Gray-Scott model. We also refer to this paper for more details on the 'Poincaré map approach' sketched in the beginning of this subsection. An analysis similar to that of [22] can be done for the generalized Gierer-Meinhardt equation by increasing $\mu$ from $\mathcal{O}(1)$ to $\mathcal{O}\left(1 / \varepsilon^{4}\right)$. Note that one has to adapt the scalings in (1.1) as soon as $\mu$ becomes $\gg 1$. It then follows that increasing $\mu$ to $\mathcal{O}\left(1 / \varepsilon^{4}\right)$ is equivalent to making $\varepsilon$ an $\mathcal{O}(1)$ parameter. We refer to [9] for the details of this scaling analysis.

2.3. Multi-circuit orbits homoclinic to $\mathcal{M}$. So far, we have only paid attention to homoclinic orbits to $\mathcal{M}$ that make a single circuit through the fast field. In this subsection, we follow [5] and establish the possibility of having orbits that take off from $\mathcal{M}$ and make $N>1$ loops through the fast field before touching down again on $\mathcal{M}$.

As we shall show below, the global stable and unstable manifolds of $\mathcal{M}$ intersect the hyperplane $\{q=0\}$ many times. The sets $\mathcal{I}_{ \pm 1}(\mathcal{M})$ defined above can be seen to be the first intersections of $W^{s}(\mathcal{M})$ and $W^{u}(\mathcal{M})$ with $\{q=0\}$ : an orbit $\gamma(\xi)$ with initial condition in $\mathcal{I}_{-1}(\mathcal{M})$ only follows the reduced fast flow for half a circuit and 'gets caught' by $\mathcal{M}$, i.e., it does not leave an exponentially small neighborhood of $\mathcal{M}$ anymore. Orbits that have their initial conditions in the second intersections of $W^{u, s}(\mathcal{M})$ with $\{q=0\}$, whose existence we shall show shortly, follow the fast flow for two half circuits through the fast field before settling down on $\mathcal{M}$. Hence, they each make one full circuit. We label these sets of initial conditions $\left(u^{0}, p^{0}, v_{ \pm 2}^{0}\left(u^{0}, p^{0}\right), 0\right)$ by $\mathcal{I}_{ \pm 2}(\mathcal{M})$. For initial conditions in them, $v_{ \pm 2}^{0}\left(u^{0}, p^{0}\right)$ are strictly $\mathcal{O}(\sqrt{\varepsilon})$.

Similar definitions can be given for the $n$-th intersection sets $\mathcal{I}_{ \pm n}(\mathcal{M})$. These sets are also two-dimensional manifolds. For $n$ even, $v_{ \pm n}^{0}$ is strictly $\mathcal{O}(\sqrt{\varepsilon})$, since these solutions make $n / 2$ full circuits in the fast field; while, for $n$ odd, $v_{ \pm n}^{0}$ is strictly $\mathcal{O}(1)$ (and $\mathcal{O}(\varepsilon)$ close to the intersection of the corresponding unperturbed homoclinic orbit of (2.1) with $\{q=0\})$, since these solutions make a half-integer number of circuits in the fast field. Below, we will show that all $\mathcal{I}_{ \pm n}(\mathcal{M})$ exist. Finally, we will show that $\mathcal{I}_{-m}(\mathcal{M}) \cap \mathcal{I}_{n}(\mathcal{M})$ for all $m+n$ even, and it is precisely in these intersections that the orbits homoclinic to $\mathcal{M}$ lie that make $\frac{m+n}{2}$ full circuits through the fast field of (1.6). Note that intersections with $m+n$ odd are ruled out due to the locations of $v_{-m}^{0}$ and $v_{n}^{0}$, since one of these is strictly $\mathcal{O}(\sqrt{\varepsilon})$, while the other is strictly $\mathcal{O}(1)$.

We first establish that the curves $\mathcal{I}_{ \pm n}(\mathcal{M})$ exist for all $n>1$ (if (1.2) holds), focusing on the case of $\mathcal{I}_{+n}(\mathcal{M})$, since the case of $\mathcal{I}_{-n}(\mathcal{M})$ may be done similarly. The plane $\left\{p^{0}=0\right\}$ separates $\mathcal{I}_{+1}(\mathcal{M})$ into two parts. The strategy then is to determine the fate of initial conditions on $\mathcal{I}_{+1}(\mathcal{M})$. We will see that there are 'wrong' and 'right' components of $\mathcal{I}_{+1}(\mathcal{M})$ such that orbits with initial conditions in the 'wrong' part of $\mathcal{I}_{+1}(\mathcal{M})$ are 'outside' the three-dimensional manifold $W^{s}(\mathcal{M})$ and follow the 
unbounded part of the integrable flow (2.1) in forward 'time' $\xi$. Hence, they do not return to $\{q=0\}$. On the other hand, orbits with initial conditions in the 'right' part of $\mathcal{I}_{+1}(\mathcal{M})$ are 'inside' the three-dimensional manifold $W^{s}(\mathcal{M})$ and follow the bounded part of the integrable flow (2.1) in forward 'time' $\xi$. Therefore, these will be the ones of interest since there is the possibility that they can return to $\{q=0\}$.

In order to deduce which part of $\mathcal{I}_{+1}(\mathcal{M})$ does return to $\{q=0\}$, i.e., which part of $\mathcal{I}_{+1}(\mathcal{M})$ is the 'right' part, we consider an orbit $\gamma_{+1}(\xi)=$ $\left(u^{+1}(\xi), p^{+1}(\xi), v^{+1}(\xi), q^{+1}(\xi)\right)$ with $\gamma_{+1}(0)=\left(u_{+1}^{0}, p_{+1}^{0}, v_{+1}^{0}, 0\right) \in I^{+1}(\mathcal{M})$. We assume that $p_{+1}^{0}$ is strictly $\mathcal{O}(\varepsilon)$, i.e., $\gamma_{+1}(0)$ is not too close to $\mathcal{I}_{-1}(\mathcal{M})$. Thus, $\gamma_{+1}$ is at its minimal distance (strictly $\mathcal{O}(\sqrt{\varepsilon})$ ) from $\mathcal{M}$ when $\xi=\Xi=\mathcal{O}(|\log \varepsilon|)$. Then, since $\gamma_{+1}(\xi) \rightarrow \mathcal{M}$ as $\xi \rightarrow-\infty$ and since $K \equiv 0$ on $\mathcal{M}$, we see that

$$
\begin{aligned}
K\left(\gamma_{+1}(\Xi)\right) & =\int_{-\infty}^{\Xi} \dot{K}(\gamma(\xi)) d \xi \\
& =\varepsilon \frac{\alpha_{2}}{\beta_{2}+1}\left(u_{+1}^{0}\right)^{\alpha_{2}-1} p_{+1}^{0} \int_{-\infty}^{\infty}\left(v^{\text {hom }}\right)^{\beta_{2}+1} d \xi+\mathcal{O}\left(\varepsilon^{2}\right)
\end{aligned}
$$

(since $v^{\text {hom }}(\xi)$ approaches 0 exponentially fast), where we have made the same approximation as in (2.12). Thus, since $\alpha_{2}<0(1.2)$, we have

$$
K\left(\gamma_{+1}(\Xi)\right)<0 \Longleftrightarrow p_{+1}^{0}>0 .
$$

Finally, since $K<0$, we know from the definition (2.7) of $K$ that $\gamma_{+1}(\Xi)$ is 'inside' $W^{s}(\mathcal{M})$; and, also that $\gamma_{+1}(\Xi)$ intersects $\{q=0\}$ again, i.e., $\mathcal{I}_{+2}(\mathcal{M})$ is nonempty. Correspondingly, the above argument shows that if $p_{+1}^{0}<0$, then $K>0$ and the orbit $\gamma_{+1}(\Xi)$ is 'outside' $W^{s}(\mathcal{M})$. Hence, it cannot intersect the hyperplane $\{q=$ $0\}$ again. The same argument in backwards 'time' yields that orbits $\gamma_{-1}(\xi)$ with $\gamma_{-1}(0)=\left(u_{-1}^{0}, p_{-1}^{0}, v_{-1}^{0}, 0\right) \in I^{-1}(\mathcal{M})$ intersect $\{q=0\}$ again when $p_{-1}^{0}<0$ (but not when $\left.p_{-1}^{0}>0\right)$. Thus, both $\mathcal{I}_{ \pm 2}(\mathcal{M})$ exist.

The above argument may be extended to show that all $\mathcal{I}_{ \pm n}(\mathcal{M})$ exist, and we denote the points in these sets by $\left(u_{ \pm n}^{0}, p_{ \pm n}^{0}, v_{ \pm n}^{0}\left(u_{ \pm n}^{0}, p_{ \pm n}^{0}\right), 0\right)$, respectively. Moreover, the same argument can be applied to any solution of (1.6) with an initial condition in $\{q=0\}$ that is at least $\mathcal{O}(\varepsilon)$ close to $W^{s}(\mathcal{M})$ or $W^{u}(\mathcal{M})$. Only the orbits with positive $p$-coordinate are 'inside' $W^{s}(\mathcal{M})$ and will thus intersect $\{q=0\}$ again in forward 'time' $(=\xi)$; the $p$-coordinate must be negative for a next intersection with $\{q=0\}$ in backward time.

Next, we show that the intersections $\mathcal{I}_{+2}(\mathcal{M}) \cap \mathcal{I}_{-2}(\mathcal{M})$, and their higher order equivalents, exist. Orbits with initial conditions in $\mathcal{I}_{ \pm 2}(\mathcal{M})$ can also be approximated by $v^{\text {hom }}(\xi)(2.1)$ to leading order, since both circuits must be $\mathcal{O}(\varepsilon)$ close to $v^{\text {hom }}(\xi)$. Thus, to leading order,

$$
\Delta K=2 \varepsilon \frac{\alpha_{2}}{\beta_{2}+1}\left(u_{+1}^{0}\right)^{\alpha_{2}-1} p_{+1}^{0} \int_{-\infty}^{\infty}\left(v^{\text {hom }}\right)^{\beta_{2}+1} d \xi,
$$

and we find that the $p$-coordinate $p^{0}$ of the initial condition must also be 0 , to leading order, for a two-circuit homoclinic orbit with initial conditions in $\mathcal{I}_{+2}(\mathcal{M}) \cap \mathcal{I}_{-2}(\mathcal{M})$. Moreover, not only is $p^{0}=0$ to leading order, but $p^{0} \equiv 0$ exactly, since the reversibility symmetry (1.7) implies that the homoclinic orbits with initial conditions $p^{0}=0$ in $\mathcal{I}_{+2}(\mathcal{M}) \cap \mathcal{I}_{-2}(\mathcal{M})$ are also symmetric, just as we saw for the one-circuit orbits. That is, we have shown

$$
\mathcal{I}_{+2}(\mathcal{M}) \cap \mathcal{I}_{-2}(\mathcal{M}) \subset\{p=0\} \text { exactly }
$$


Finally, the same argument may be repeated inductively to show that

$$
\mathcal{I}_{+n}(\mathcal{M}) \cap \mathcal{I}_{-n}(\mathcal{M}) \subset\{p=0\} \text { for all } n=\mathcal{O}(1) \text { exactly }
$$

So far, however, it is not yet clear for which pairs $m \neq n$ with $m+n$ even,

$$
\mathcal{I}_{+n}(\mathcal{M}) \cap\{p=0\} \neq \emptyset \quad \text { and } \quad \mathcal{I}_{-m}(\mathcal{M}) \cap\{p=0\} \neq \emptyset
$$

As shown above, only those orbits with initial conditions in $\mathcal{I}_{+1}(\mathcal{M}) \cup\{p>0\}$ can 'build' $\mathcal{I}_{+2}(\mathcal{M})$. The main question then is: Can (at least) one of these orbits satisfy $p(\xi)=0$ at its second intersection with $\{q=0\}$ ?

We begin by calculating the change in $p$ during the half-circuit from $\{q=0\}$ back to $\{q=0\}$. Note that a similar computation was already done at the beginning of section 2.2. We consider the orbit $\gamma_{+1}(\xi)=\left(u_{+1}(\xi), p_{+1}(\xi), v_{+1}(\xi), q_{+1}(\xi)\right)$ with $\gamma_{+1}(0)=\left(u_{+1}^{0}, p_{+1}^{0}, v_{+1}^{0}, 0\right) \in I^{+1}(\mathcal{M})$, where now $p_{+1}^{0}=\varepsilon \tilde{p}^{0}>0$ with $\tilde{p}^{0}$ strictly $\mathcal{O}(1)$. Let $\Xi(=\mathcal{O}(|\log \varepsilon|))$ be such that $\gamma_{+1}(\Xi) \in \mathcal{I}_{+2}(\mathcal{M}) \subset\{q=0\}$. We define $\Delta p$ by $p^{+1}(\Xi) \stackrel{\text { def }}{=} \varepsilon \tilde{p}^{0}+\Delta p$. Hence, by the second component of $(1.6)$,

$$
\Delta p^{+1}\left(u^{0}, \varepsilon \tilde{p}^{0}\right)=-\varepsilon \int_{0}^{\Xi} u^{\alpha_{1}} v^{\beta_{1}} d \xi+\mathcal{O}\left(\varepsilon^{3}|\log \varepsilon|\right) ;
$$

note that $\Delta p^{+1}\left(u^{0}, \varepsilon \tilde{p}^{0}\right)$ is finite (1.2). Thus,

$$
\Delta p^{+1}\left(u^{0}, \varepsilon \tilde{p}^{0}\right)=-\varepsilon\left(u^{0}\right)^{\alpha_{1}} \int_{0}^{\infty}\left(v^{\text {hom }}(\xi)\right)^{\beta_{1}} d \xi+\mathcal{O}\left(\varepsilon^{2}\right),
$$

As a consequence, the $p$-coordinate of $\gamma_{+1}(\Xi) \in \mathcal{I}_{+2}(\mathcal{M})$ is given to leading order by

$$
\varepsilon\left[\tilde{p}^{0}-\left(u^{0}\right)^{\alpha_{1}} \int_{0}^{\infty}\left(v^{\text {hom }}(\xi)\right)^{\beta_{1}} d \xi\right] \text { with } \tilde{p}^{0}>0 .
$$

This expression can change sign (since $u^{0}>0$ and $v^{\text {hom }}(\xi)>0$ ). By a similar argument for the general case, we conclude that all intersections $\mathcal{I}_{+n}(\mathcal{M}) \cap \mathcal{I}_{-n}(\mathcal{M})$ exist and satisfy (2.19). Moreover, by following the fast flow for $j$ half circuits, we see that all $\mathcal{I}_{n+j}(\mathcal{M}) \cap \mathcal{I}_{-n+j}(\mathcal{M})$ exist, although these sets are not subsets of $\{p=0\}$, since $\Delta p \neq 0$, by $(2.22)$ or its equivalents. Summarizing,

$$
\mathcal{I}_{+n}(\mathcal{M}) \cap \mathcal{I}_{-m}(\mathcal{M}) \neq \emptyset \text { for all } n+m \text { even and } m, n=\mathcal{O}(1) .
$$

2.4. Take off and touch down curves. So far, we have focused on the dynamics in the fast field of (1.6), and for every $\mathcal{O}(1) N>0$, we have constructed a one-parameter family of multi-circuit orbits homoclinic to $\mathcal{M}$. We now turn our attention for each $N \geq 1$ to locating special pairs of curves on $\mathcal{M}$ that are essential for determining the slow segments of these same multi-circuit orbits. In particular, we will need the ideas developed in [11].

Let $\gamma_{N}(\xi)$ be an $N$-circuit orbit homoclinic to $\mathcal{M}$, of the type whose existence has been shown in the previous subsection, with $\gamma_{N}(0) \in \mathcal{I}_{N}(\mathcal{M}) \cap \mathcal{I}_{-N}(\mathcal{M}) \subset$ $\{p=0\}$. By geometrical singular perturbation theory (see [11] and [18]), there are two orbits $\gamma_{+N}^{\text {slow }}=\gamma_{+N}^{\text {slow }}\left(\xi ;\left(u_{+N}^{0}, p_{+N}^{0}\right)\right) \subset \mathcal{M}$ and $\gamma_{-N}^{\text {slow }}\left(\xi ;\left(u_{-N}^{0}, p_{-N}^{0}\right)\right) \subset$ $\mathcal{M}$, respectively (where $\left.\gamma_{ \pm N}\left(0 ;\left(u_{ \pm N}^{0}, p_{ \pm N}^{0}\right)\right)=\left(u_{ \pm N}^{0}, p_{ \pm N}^{0}\right) \in \mathcal{M}\right)$, such that $\left\|\gamma_{N}(\xi)-\gamma_{+N}^{\text {slow }}\left(\xi ;\left(u_{+N}^{0}, p_{+N}^{0}\right)\right)\right\|$ is exponentially small for $\xi>0$ with $\xi \geq \mathcal{O}\left(\frac{1}{\varepsilon}\right)$ and 
$\left\|\gamma_{N}(\xi)-\gamma_{-N}^{\text {slow }}\left(\xi ;\left(u_{-N}^{0}, p_{-N}^{0}\right)\right)\right\|$ is exponentially small for $\xi<0$ with $-\xi \geq \mathcal{O}\left(\frac{1}{\varepsilon}\right)$. As a consequence,

$$
d\left(\gamma_{N}(\xi), \mathcal{M}\right)=\mathcal{O}\left(e^{-\frac{k}{\varepsilon}}\right) \text { for }|\xi| \geq \mathcal{O}\left(\frac{1}{\varepsilon}\right) \text { or larger, }
$$

for some $k>0$. The orbits $\gamma_{ \pm N}^{\text {slow }}\left(\xi ;\left(u_{ \pm N}^{0}, p_{ \pm N}^{0}\right)\right)$ determine the behavior of $\gamma_{N}(\xi)$ near $\mathcal{M}$. Moreover, $\gamma_{N}(\xi)$ satisfies the reversibility symmetry (1.7) by the choice of initial conditions, and thus

$$
\gamma_{-N}^{\text {slow }}\left(\xi ;\left(u_{-N}^{0}, p_{-N}^{0}\right)\right)=\gamma_{+N}^{\text {slow }}\left(-\xi ;\left(u_{+N}^{0},-p_{+N}^{0}\right)\right) .
$$

We now define the curves $T_{N}^{\text {down }} \subset \mathcal{M}$ ('touch down') and $T_{N}^{\text {off }} \subset \mathcal{M}$ ('take off') as

$$
\begin{array}{r}
T_{N}^{\text {down }}=\cup_{\gamma_{N}(0)}\left\{\left(u_{+N}^{0}, p_{+N}^{0}\right)=\gamma_{N}^{\text {slow }}\left(0 ;\left(u_{+N}^{0}, p_{+N}^{0}\right)\right)\right\}, \\
\text { and } T_{N}^{\text {off }}=\cup_{\gamma_{N}(0)}\left\{\left(u_{+N}^{0},-p_{+N}^{0}\right)\right\},
\end{array}
$$

where the unions are over all $\gamma_{N}(0) \in \mathcal{I}_{N}(\mathcal{M}) \cap \mathcal{I}_{-N}(\mathcal{M}) \subset\{p=0\} \cap\{q=0\}$. For each $N=1,2, \ldots$, the take off set $T_{N}^{\text {off }}$ (respectively, the touch down set $T_{N}^{\text {down }}$ ) is the collection of base points of all of the Fenichel fibers in $W^{u}(\mathcal{M})$ (respectively, $W^{s}(\mathcal{M})$ ) that have points in the transverse intersection of $W^{u}(\mathcal{M})$ and $W^{s}(\mathcal{M})[10,11,18,19$, 20].

Detailed asymptotic information about the locations of $T_{N}^{\text {off }}$ and $T_{N}^{\text {down }}$ can be obtained explicitly by determining the relations between $\gamma_{N}(0)=\left(u^{0}, 0, v^{0}, 0\right)$ and $\left(u_{+N}^{0}, p_{+N}^{0}, 0,0\right)$. First, we observe that $\dot{p}=\mathcal{O}\left(\varepsilon^{3}\right)$ on $\mathcal{M}(1.6)$, thus, the $p$-coordinate of $\gamma_{+N}^{\text {slow }}$ remains constant to leading order during the fast excursions of $\gamma_{N}(\xi)$. Therefore, $p_{+N}^{0}$ is completely determined (to leading order) by the accumulated change in $p$ of $\gamma_{N}(\xi)$ during its 'time' $\xi>0$ in the fast field. These changes have already been calculated for $N=1$ in (2.21) and (2.22). For $N>1$, the calculation is exactly the same, except for the fact that $\gamma_{N}(\xi)$ now makes $N$ half circuits before 'touching down' on $\mathcal{M}$ :

$$
p_{+N}^{0}=-\varepsilon N\left(u^{0}\right)^{\alpha_{1}} \int_{0}^{\infty}\left(v^{\text {hom }}(\xi)\right)^{\beta_{1}} d \xi+\mathcal{O}\left(\varepsilon^{2}\right),
$$

From the first component of (1.6) and the fact that $p=\mathcal{O}(\varepsilon)$, we also conclude that $u_{+N}^{0}=u^{0}$ to leading order. Thus, we find

$$
\begin{array}{r}
T_{N}^{\text {down }}:\left\{p \stackrel{\text { def }}{=} p_{N}^{\text {down }}(u)=-\varepsilon N u^{\alpha_{1}} \int_{0}^{\infty}\left(v^{\text {hom }}(\xi)\right)^{\beta_{1}} d \xi+\mathcal{O}\left(\varepsilon^{2}\right)\right\} \\
\text { and } T_{N}^{\text {off }}:\left\{p=-p_{N}^{\text {down }}(u)\right\},
\end{array}
$$

where $v^{\text {hom }}(\xi)=v^{\text {hom }}(\xi ; u)$, the homoclinic solution of $(2.1)$.

To more fully determine the behavior of $p_{N}^{\text {down }}$ as a function of $u$, we introduce $w^{\text {hom }}=w^{\text {hom }}\left(\xi ; \beta_{2}\right) \geq 0$, which is the (positive) homoclinic solution of a rescaled version of $(2.1)$ :

$$
\ddot{w}=w-w^{\beta_{2}} .
$$

Without loss of generality, we take the solutions to be parameterized such that $w^{\text {hom }}(\xi)$ is symmetric with respect to $\xi=0$. Thus,

$$
v^{\text {hom }}(\xi ; u)=v^{\text {hom }}\left(\xi ; u, \alpha_{2}, \beta_{2}\right)=\left(u^{\alpha_{2}}\right)^{\frac{1}{1-\beta_{2}}} w^{\text {hom }}\left(\xi ; \beta_{2}\right),
$$




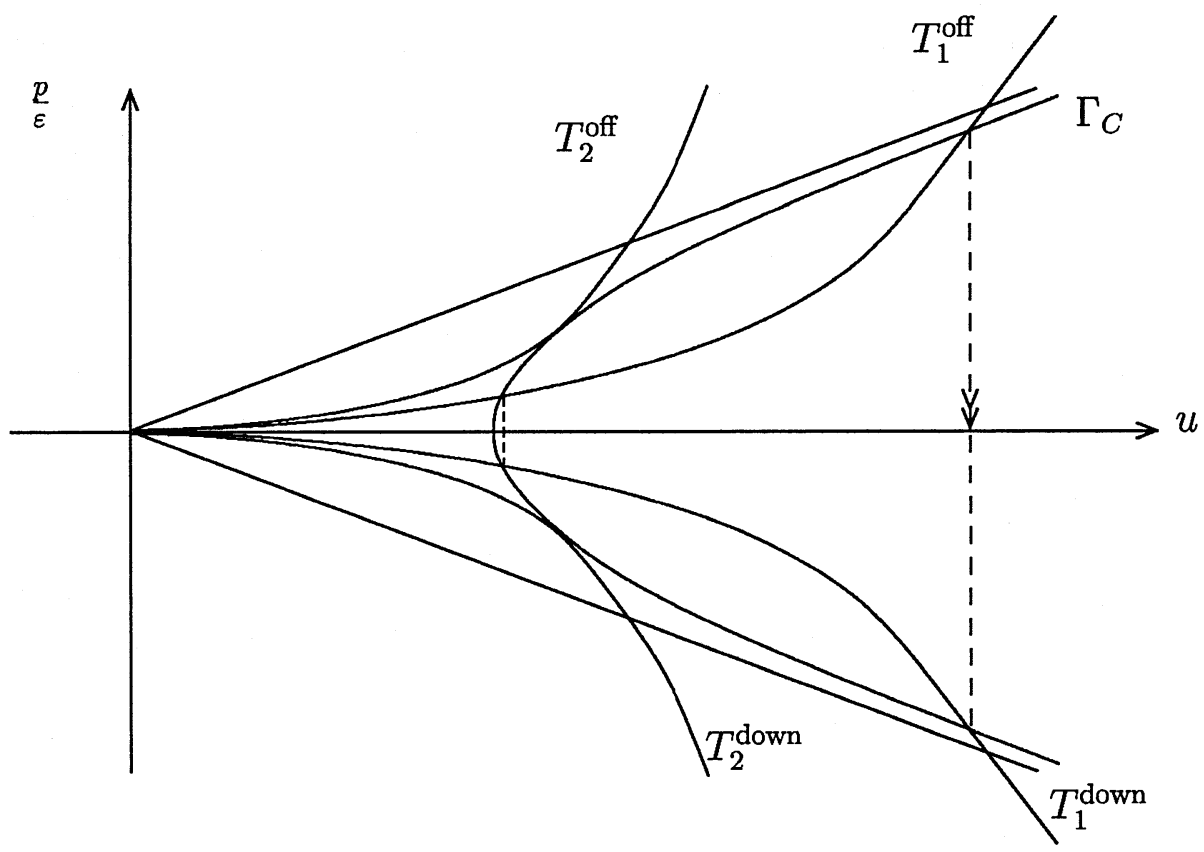

FIG. 2.1. A representation of the relevant part of the (linear) flow on $\mathcal{M}$ (for typical parameter values) and the curves $T_{1}^{\text {off }}, T_{1}^{\text {down }}, T_{2}^{\text {off }}$ and $T_{2}^{\text {down }}$. Note that the orbit $\Gamma_{C}$ has been chosen such that $C=C_{2}^{\mathrm{SN}}$.

which yields

$$
\int_{-\infty}^{\infty}\left(v^{\text {hom }}(\xi)\right)^{\beta_{1}} d \xi=2 \int_{0}^{\infty}\left(v^{\text {hom }}(\xi)\right)^{\beta_{1}} d \xi=u^{\frac{-\alpha_{2} \beta_{1}}{\beta_{2}-1}} W\left(\beta_{1}, \beta_{2}\right)
$$

where

$$
W\left(\beta_{1}, \beta_{2}\right)=\int_{-\infty}^{\infty}\left(w^{\text {hom }}\left(\xi ; \beta_{2}\right)\right)^{\beta_{1}} d \xi
$$

We can now rewrite (2.30) to leading order as

$(2.34) T_{N}^{\text {down,off }}:\left\{p= \pm p_{N}^{\text {down }}(u)\right\}$ with $p_{N}^{\text {down }}(u)=-\frac{1}{2} N \varepsilon u^{1+\frac{D}{\beta_{2}-1}} W\left(\beta_{1}, \beta_{2}\right)$,

with $D>0$ (1.2), (1.3). Note that the higher order corrections, which are not needed here, can be obtained by a straightforward asymptotic approximation scheme, see [7].

In Figure 2.1, we have plotted the curves $T_{N}^{\text {down,off }}$ for a few values of $N$ superimposed onto the linear flow on $\mathcal{M}$ given by (2.2). Since $D>0$ and $\beta_{2}>1$, the curve $T_{N}^{\text {off }}$ given by (2.34) to leading order is tangent to the $u$-axis for each $N$. Thus, by (2.3), both $T_{N}^{\text {off }} \cap \ell^{u}$ and $T_{N}^{\text {down }} \cap \ell^{s}$ consist for all $N$ of one uniquely determined point in the half-plane $\{u>0\}$-coordinate. By the symmetry (1.7), both intersections have exactly the same $u$ coordinate, that is given to leading order by

$$
u_{N}^{\text {hom }}(\mu)=\left[\frac{2 \sqrt{\mu}}{N W\left(\beta_{1}, \beta_{2}\right)}\right]^{\frac{\beta_{2}-1}{D}}
$$


It was proven in [5] that there corresponds a unique homoclinic orbit $\gamma_{N}^{\text {hom }}(\xi)$ to the saddle point $S$ to any pair of intersections (with positive $u$-coordinates) $\left\{T_{N}^{\text {down }} \cap\right.$ $\left.\ell^{u}, T_{N}^{\text {off }} \cap \ell^{s}\right\}=\Gamma_{0} \cap\left(T_{N}^{\text {down }} \cup T_{N}^{\text {off }}\right)(2.4)$. This result is formulated as Theorem 1.1 in the Introduction.

The periodic solutions to be constructed in the next section are exponentially close to (parts of) the (multi-pulse) homoclinic solutions associated to the intersections $\Gamma_{C} \cap\left(T_{N}^{\text {down }} \cup T_{N}^{\text {off }}\right)$ with $C>0$ (and $u>0$ ). Remark 2.2 below also addresses our reasons for focusing on $C>0$ here. It is a straightforward calculation to check that $\Gamma_{C} \cap\left(T_{N}^{\text {down }} \cup T_{N}^{\text {off }}\right) \neq \emptyset$ for $0 \leq C \leq C_{N}^{\mathrm{SN}}(\mu)$ where, to leading order,

$$
C_{N}^{\mathrm{SN}}(\mu)=\frac{D}{\beta_{2}-1+D} \mu\left(u_{N}^{\mathrm{SN}}(\mu)\right)^{2} \text { with } u_{N}^{\mathrm{SN}}(\mu)=\left[\frac{\beta_{2}-1}{\beta_{2}-1+D}\right]^{\frac{\beta_{2}-1}{2 D}} u_{N}^{\text {hom }}(\mu)
$$

(2.35): Here, $u_{N}^{\mathrm{SN}}(\mu)<u_{N}^{\text {hom }}(\mu)$ is the $u$-coordinate of $\Gamma_{C} \cap\left(T_{N}^{\text {down }} \cup T_{N}^{\text {off }}\right)$ at $C=C_{N}^{\mathrm{SN}}(\mu)$, the value of $C$ at which $\Gamma_{C}$ is tangent to $T_{N}^{\text {down }} \cup T_{N}^{\text {off }}$. Note that $\lim _{N \rightarrow \infty} C_{N}^{\mathrm{SN}}=0$.

We conclude that for $0<C<C_{N}^{\mathrm{SN}}(\mu), \Gamma_{C} \cap\left(T_{N}^{\text {down }} \cup T_{N}^{\text {off }}\right) \cap\{u>0\}$ consists of 4 points, i.e.,

$$
\begin{aligned}
\Gamma_{C} \cap\left(T_{N}^{\text {down }} \cup T_{N}^{\text {off }}\right) \cap\{u>0\} & =P_{A_{N}}^{ \pm}(C) \cup P_{B_{N}}^{ \pm}(C) \\
& =\left\{\left(u_{A_{N}}^{\text {per }}(C), \pm p_{A_{N}}^{\text {per }}(C)\right),\left(u_{B_{N}}^{\text {per }}(C), \pm p_{B_{N}}^{\text {per }}(C)\right)\right\}
\end{aligned}
$$

where

$$
0<u_{B_{N}}^{\text {per }}(C)<u_{N}^{\mathrm{SN}}(C)<u_{A_{N}}^{\text {per }}(C)<u_{N}^{\text {hom }}
$$

see Figure 2.1. Note that $\lim _{C \downarrow 0} u_{B_{N}}^{\text {per }}(C)=0$ and $\lim _{C \downarrow 0} u_{A_{N}}^{\text {per }}(C)=u_{N}^{\text {hom }}$.

REMARK 2.2. For $C<0$, the intersections $\Gamma_{C} \cap\left(T_{N}^{\text {down }} \cup T_{N}^{\text {off }}\right)$ correspond to homoclinic orbits to $\mathcal{M}$ of which the $u$-coordinate becomes negative and unbounded for sufficiently large $|\xi|$. Such solutions are unrealistic as solutions of the PDE (1.1) and are not considered in any further detail here.

\section{Singular, periodic, multi-pulse solutions.}

3.1. The fundamental periodic solutions. The first extension of the existence result on homoclinic solutions, Theorem 1.1 (originally from [5]), is a result on the existence of so-called 'fundamental' periodic orbits. For any $N \geq 1$ of $\mathcal{O}(1)$ there are two one-parameter families of such orbits. The $N$-pulse homoclinic solutions of Theorem 1.1 can be interpreted as being on the boundary of one of these families (see Corollary 3.2).

THEOREM 3.1: THE FUndAMENTAL PERIOdiC SOlUtions. Let $\left(\alpha_{1}, \alpha_{2}, \beta_{1}, \beta_{2}, \mu\right)$ satisfy (1.2). Then, for any $N \geq 1$ with $N=\mathcal{O}(1)$ and $\varepsilon>0$ small enough, (1.6) possesses two one-parameter families of periodic orbits, $\gamma_{A_{N}}^{\mathrm{per}}(\xi)$ and $\gamma_{B_{N}}^{\mathrm{per}}(\xi)$, with positive $u, v$ coordinates. For each $N$, each solution, $\gamma_{A_{N}}^{\text {per }}(\xi)=\gamma_{A_{N}}^{\text {per }}(\xi ; C), \gamma_{B_{N}}^{\text {per }}(\xi)=$ $\gamma_{B_{N}}^{\text {per }}(\xi ; C)$, respectively, consists of a slow piece on which it is exponentially close to a part of an orbit $\Gamma_{C}$ (2.4) on $\mathcal{M}$ with $0<C<C_{N}^{\mathrm{SN}}(\mu)$ (2.36) as well as a fast jump in which it makes $N$ circuits through the fast space during which it remains at least $\mathcal{O}(\sqrt{\varepsilon})$ away from $\mathcal{M}$.

In Figure 3.1, two examples of fundamental periodic orbits are sketched in the 4-dimensional phase space of (1.6). The periodic patterns $\left(U_{A_{N}}^{\mathrm{per}}(x), V_{A_{N}}^{\mathrm{per}}(x)\right)$ and 


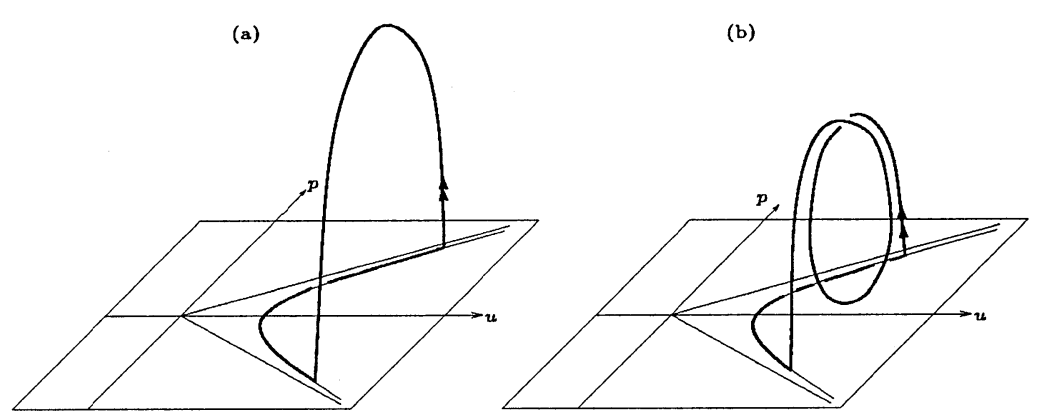

FIG. 3.1. Two schematic illustrations of fundamental periodic orbits in the four-dimensional phase space associated to (1.6). Note that the direction perpendicular to the $\{u, p\}$-plane $\mathcal{M}$ is represented by $v$. (a) An orbit $\gamma_{A_{1}}^{\text {per }}(\xi ; C)$; (b) an orbit $\gamma_{A_{2}}^{\text {per }}(\xi ; C)$ that makes two circuits through the fast field in between take off from and touch down on $\mathcal{M}$.

$\left(U_{B_{N}}^{\text {per }}(x), V_{B_{N}}^{\text {per }}(x)\right)$ of the PDE (1.4) associated to the orbits $\gamma_{A_{N}}^{\text {per }}(\xi)$ and $\gamma_{B_{N}}^{\text {per }}(\xi)$, respectively, have the following structure. They consist of long intervals on which the $U$-components vary slowly and on which the $V$-components are exponentially small (these parts correspond to the slow pieces of the orbits $\gamma_{A_{N}}^{\text {per }}(\xi)$ and $\gamma_{B_{N}}^{\text {per }}(\xi)$ near $\mathcal{M})$. Moreover, these long intervals are interspersed with narrow intervals in which the $V$-components have $N$ distinct pulses (the $N$ fast jumps of the orbits $\gamma_{A_{N}}^{\text {per }}(\xi)$ and $\gamma_{B_{N}}^{\text {per }}(\xi)$ ), and in which the $U$-components $U_{A_{N}}^{\text {per }}(x)$ and $U_{B_{N}}^{\text {per }}(x)$ are constant to leading order. See Figure 1.1a for a finite part of a (numerically) stable pattern $\left(U_{A_{1}}^{\text {per }}(x), V_{A_{1}}^{\text {per }}(x)\right)$, i.e., $N=1$. The structure of a pattern with pulses of $A_{N}$-type has been schematically illustrated in Figure 1.2 for $N=2$ and $N=3$. Note that the amplitudes of both $U_{A_{N}}^{\text {per }}(x)$ and $V_{A_{N}}^{\text {per }}(x)$ are larger than the amplitudes of $U_{B_{N}}^{\text {per }}(x)$ and $V_{B_{N}}^{\text {per }}(x)$ by $(2.38)$ and $(2.31)$.

The proof of Theorem 3.1 is very similar to the proof of a theorem on the existence of (stationary) spatially periodic patterns in the Gray-Scott model (see [7]; note, however, that only the case $N=1$ has been considered in [7]). However, in the Gray-Scott the counterpart of the intersections $\Gamma_{C} \cap\left(T_{N}^{\text {down }} \cup T_{N}^{\text {off }}\right)$ consist of only two (symmetrical) points: the take off point of the periodic orbit and the touch down point of the periodic orbit. As we have seen in the previous section, for any $N \geq 1$ and $0<C<C_{N}^{\mathrm{SN}}(2.36)$ there are two pairs of take off/touch down points in the GiererMeinhardt case, $\left(P_{A_{N}}^{+}(C), P_{A_{N}}^{-}(C)\right),\left(P_{B_{N}}^{+}(C), P_{B_{N}}^{-}(C)\right) \in \Gamma_{C}$ (2.37). This explains the existence of two families of periodic orbits (for each given $N$ ). This feature of the Gierer-Meinhardt model enables us to construct countably many classes of periodic solutions of 'mixed' type, as we shall show in the next subsection. The construction of these 'mixed' solutions is based on the 'skeleton' spanned by the fundamental solutions described by Theorem 3.1.

Proof of Theorem 3.1. Choose an $N>0$ and a $C \in\left(0, C_{N}^{\mathrm{SN}}\right)(2.36)$. We consider an exponentially small interval $I_{A, B}$ on the halfline $\subset\{p=q=0\}$, perpendicular to $\mathcal{M}$ with $u \equiv u^{\min }(C)(2.6)$ and $v=v^{0} \in\left(b_{\ell} e^{-1 / \varepsilon^{2}}, b_{u} e^{-1 / \varepsilon^{2}}\right)$ for certain constants $0<b_{\ell}<b_{u}$. Note that the choice of the exponent $-1 / \varepsilon^{2}$ is determined by the unstable eigenvalue $\lambda=1$ of the fast reduced limit problem (2.1).

Any solution $\gamma(\xi)$ of (1.6) with initial conditions on $I_{A, B}$, i.e. $\gamma(0)=$ $\left(u^{\min }(C), 0, v^{0}, 0\right)$ will remain $\mathcal{O}\left(e^{-1 / \varepsilon^{2}}\right)$ close to $\mathcal{M}$ for $\mathcal{O}\left(1 / \varepsilon^{2}\right)$ time $\xi$, during which it follows the solution $\Gamma_{C}$ on $\mathcal{M}(2.4)$ at an $\mathcal{O}\left(e^{-1 / \varepsilon^{2}}\right)$ distance. Hence, the $u$-coordinate of $\gamma(\xi)$ will change by an $\mathcal{O}(1)$ amount before $\gamma(\xi)$ takes off from $\mathcal{M} ; \gamma(\xi)$ remains 
$C^{1}-\mathcal{O}\left(e^{-1 / \varepsilon^{2}}\right)$ close to $W^{u}(\mathcal{M})([19],[27],[26])$ after take off. We can even be more precise. Define $W^{u}\left(\Gamma_{C}\right) \subset W^{u}(\mathcal{M})$ as the two-dimensional submanifold of orbits asymptotic to $\mathcal{M}$ (as $\xi \rightarrow-\infty)$ that take off from $\Gamma_{C}$ (see section 2.4). It follows that $\gamma(\xi)$ is $C^{1}-\mathcal{O}\left(e^{-1 / \varepsilon^{2}}\right)$ close to $W^{u}\left(\Gamma_{C}\right)$.

The manifold $W^{u}\left(\Gamma_{C}\right) \subset W^{u}(\mathcal{M})$ intersects the hyperplane $\{q=0\}$ transversally, and $W^{u}\left(\Gamma_{C}\right) \cap\{q=0\} \subset \mathcal{I}_{+1}$ (section 2.2). It follows from the $\Delta p$ analysis in section 2.3 that the $p$-coordinate of the first intersection (i.e. $N=1) W^{u}\left(\Gamma_{C}\right) \cap\{q=0\}$ is positive for orbits in $W^{u}\left(\Gamma_{C}\right)$ that take off from points on $\Gamma_{C}$ in between $P_{B_{1}}^{+}(C)$ and $P_{A_{1}}^{+}(C)(2.37)$ - i.e. the points on $\mathcal{M}$ 'above' $T_{1}^{\text {off }}$ (Figure 2.1 ) - while $W^{u}\left(\Gamma_{C}\right) \cap\{q=$ $0\}$ has negative $p$-coordinates for the other orbits in $W^{u}\left(\Gamma_{C}\right)$. We know by the reversibility symmetry (1.7) that the $p$-coordinates change sign exactly at the two homoclinic orbits associated to $\Gamma_{C} \cap\left(T_{1}^{\text {down }} \cup T_{1}^{\text {off }}\right)$ that intersect $\{q=0\}$ in $\mathcal{I}_{+1} \cap \mathcal{I}_{-1}$. The $\Delta p$ calculations in section 2.3 also imply that only the orbits that take off from $\Gamma_{C}$ in between $P_{B_{1}}^{+}(C)$ and $P_{A_{1}}^{+}(C)$ will have a second intersection with $\{q=0\} \in \mathcal{I}_{+2}$ (by definition, section 2.3), and that the $p$-coordinate of this second intersection of $W^{u}\left(\Gamma_{C}\right)$ with $\{q=0\}$ again changes sign twice (where the zeroes again correspond to the 2 homoclinic orbits associated to $\Gamma_{C} \cap\left(T_{2}^{\text {down }} \cup T_{2}^{\text {off }}\right)$ and to $\left.\mathcal{I}_{+2} \cap \mathcal{I}_{-2}\right)$. Only those orbits that have a positive $p$-coordinate at their second intersection with $\{q=0\}$ will return to $\{q=0\}$ for a third intersection $\left(\in \mathcal{I}_{+3}\right)$. These are, by construction, the orbits that take off from $\mathcal{M}$ on points of $\Gamma_{C}$ in between $P_{B_{2}}^{+}(C)$ and $P_{A_{2}}^{+}(C)$.

By the principle of finite induction, this argument can be iterated: the $p$ coordinate of the $N$-th intersection of $W^{u}\left(\Gamma_{C}\right)$ with $\{q=0\}\left(\in \mathcal{I}_{+N}\right)$ changes sign twice. Only orbits that take off from $\mathcal{M}$ on points of $\Gamma_{C}$ in between $P_{B_{N}}^{+}(C)$ and $P_{A_{N}}^{+}(C)$ will have an $N+1$-th intersection with $\{q=0\}$. Note that this iteration is consistent: for all $N \geq 1$, the part of $\Gamma_{C}$ in between $P_{B_{N+1}}^{+}(C)$ and $P_{A_{N+1}}^{+}(C)$ is a subset of that between $P_{B_{N}}^{+}(C)$ and $P_{A_{N}}^{+}(C)$.

Now consider the two-dimensional manifold $\mathcal{L}_{A, B}$ generated by solutions of (1.6) with initial conditions on $I_{A, B}$. This manifold is, by construction, exponentially close to $W^{u}\left(\Gamma_{C}\right)$. The constant $b_{u}$ can be chosen such that the orbit through the 'upper' boundary of $\mathcal{L}_{A, B}$ takes off from $\mathcal{M}$ near a point on $\Gamma_{C}$ with a $u$ coordinate that is smaller than $u_{B_{1}}^{\text {per }}(C)$, the $u$ coordinate of $P_{B_{1}}^{+}(C)(2.37)$; likewise, $b_{\ell}$ can be chosen such that the 'lower' boundary of $\mathcal{L}_{A, B}$ takes off from $\mathcal{M}$ near a point on $\Gamma_{C}$ with a $u$ coordinate that is larger than $u_{A_{1}}^{\mathrm{per}}(C)>u_{B_{1}}^{\mathrm{per}}(C)(2.38)$, the $u$ coordinate of $P_{A_{1}}^{+}(C)$. Since $\mathcal{L}_{A, B} \cap\{q=0\}$ is exponentially close to $W^{u}\left(\Gamma_{C}\right) \cap\{q=0\} \cap \mathcal{I}_{+N}$, we may therefore conclude that there are two solutions, $\gamma_{A_{N}}^{\text {per }}(\xi)$ and $\gamma_{B_{N}}^{\text {per }}(\xi)$, of (1.6) with initial conditions on $I_{A, B}$ that have an $N$-th intersection with $\{q=0\}$ with a $p$-coordinate that is identically zero. Note that the orbits $\gamma_{A_{N}}^{\mathrm{per}}(\xi ; C)$ and $\gamma_{B_{N}}^{\mathrm{per}}(\xi ; C)$ take off from $\mathcal{M}$ exponentially close to the points $P_{A_{N}}^{+}, P_{B_{N}}^{+}(C) \in \Gamma_{C}$, respectively.

We use once again the reversibility symmetry $(1.7)$ to extend the orbits $\gamma_{A_{N}}^{\text {per }}(\xi)$ and $\gamma_{B_{N}}^{\text {per }}(\xi)$ to negative $\xi$ by setting $\gamma_{A_{N}}^{\text {per }}(-\xi)=\gamma_{A_{N}}^{\text {per }}(\xi)$ and $\gamma_{B_{N}}^{\text {per }}(-\xi)=\gamma_{B_{N}}^{\text {pef }}(\xi)$. Hence, we have established that $\gamma_{A_{N}}^{\text {per }}(\xi)$ and $\gamma_{B_{N}}^{\text {per }}(\xi)$ are indeed periodic solutions of (1.6).

We state without further proof (see also section 4.3 of [7]):

Corollary 3.2: A SADDle NODE BIFURCATION OF PERIODIC ORBITS. The periodic orbits $\gamma_{A_{N}}^{\text {per }}(\xi ; C)$ and $\gamma_{B_{N}}^{\text {per }}(\xi ; C)$ merge in a saddle node bifurcation of periodic orbits as $C \uparrow C_{N}^{\mathrm{SN}}(\mu)$. Finally, $\gamma_{A_{N}}^{\text {per }}(\xi ; C) \rightarrow \gamma_{N}^{\text {hom }}(\xi)$ (Theorem 1.1) and $\gamma_{B_{N}}^{\text {per }}(\xi ; C) \rightarrow$ $(0,0,0,0)$ as $C \downarrow 0$. 
3.2. Periodic solutions of mixed type. The fundamental periodic orbits constructed in the previous section only make one excursion through the fast field (see Figure 3.1). In this section we will show that periodic orbits can also be of 'mixed type' in the sense that a periodic orbit may make various different excursions through the fast field.

To distinguish between all possible mixed periodic orbits, we define a labeling of the possible 'jumps' through the fast field. A jump of an orbit from $\mathcal{M}$ to $\mathcal{M}$ is said to be of $A_{N}$-type, respectively $B_{M}$-type, when the orbit takes off from $\mathcal{M}$ exponentially close to a point $P_{A_{N}}^{+}(C) \in \Gamma_{C}$ (respectively $P_{B_{M}}^{+}(C) \in \Gamma_{C}$ ), makes $N$ (respectively $M$ ) circuits through the fast field, and touches down on $\mathcal{M}$ close to $P_{A_{N}}^{-}(C)\left(P_{B_{M}}^{-}(C)\right)$. The set of all possible jumps through the fast field is thus given by $\left\{A_{N}, B_{M}\right\}_{N, M \geq 1}$.

A singular periodic orbit $\gamma^{\text {per }}(\xi ; C)$ is said to be of $\sigma_{1} \sigma_{2} \ldots \sigma_{J}$-type, where $\sigma_{j} \in$ $\left\{A_{N}, B_{M}\right\}_{N, M \geq 1}, j=1,2, \ldots, J$, when it follows an orbit $\Gamma_{C} \subset \mathcal{M}$ at exponentially small distance, takes off at $P_{\sigma_{1}}^{+}(C)$, makes a jump of $\sigma_{1}$-type through the fast field, touches down on $\mathcal{M}$ near $P_{\sigma_{1}}^{-}(C)$, follows $\Gamma_{C}$ again, takes off at $P_{\sigma_{2}}^{+}(C)$ for a $\sigma_{2^{-}}$ jump, touches down on $\mathcal{M}$, makes a $\sigma_{3}$-jump etc.. After the $\sigma_{J}$-jump, $\gamma^{\text {per }}(\xi)$ touches down on $\mathcal{M}$, follows $\Gamma_{C}$ and takes off again at $P_{\sigma_{1}}^{+}(C)$ for identically the same $\sigma_{1^{-}}$ jump as the one that started the series of jumps. Such an orbit $\gamma^{\text {per }}(\xi)$ is denoted by $\gamma_{\sigma_{1} \sigma_{2} \ldots \sigma_{J}}^{\text {per }}(\xi ; C)$. The solution $\left(U_{\sigma_{1} \sigma_{2} \ldots \sigma_{J}}^{\text {per }}(x), V_{\sigma_{1} \sigma_{2} \ldots \sigma_{J}}^{\text {per }}(x)\right)$ of $(1.4)$ given by the orbit $\gamma_{\sigma_{1} \sigma_{2} \ldots \sigma_{J}}^{\text {per }}(\xi ; C)$ corresponds to a pattern with a periodic sequence of pulses $\ldots \sigma_{1} \sigma_{2} \ldots \sigma_{J} \sigma_{1} \sigma_{2} \ldots \sigma_{J} \sigma_{1} \sigma_{2} \ldots \sigma_{J} \ldots$ (see Figure 1.2 for a sketch). Note that a $\sigma_{1} \sigma_{2} \ldots \sigma_{J^{-}}$ orbit is identical to a $\sigma_{2} \sigma_{3} \ldots \sigma_{J} \sigma_{1}$-orbit, a $\sigma_{3} \ldots \sigma_{J} \sigma_{1} \sigma_{2}$-orbit, etc..

The fundamental orbits constructed in Theorem 3.1, denoted by $\gamma_{\sigma}^{\text {per }}(\xi ; C)$ with $\sigma \in\left\{A_{N}, B_{M}\right\}_{N, M \geq 1}$, are thus by definition of $\sigma$-type. The simplest periodic orbits of mixed type are the $\sigma_{1} \sigma_{2}$-orbits. These orbits only make two different jumps through the fast space, one of $\sigma_{1}$ type, the other of $\sigma_{2}$-type. Note that the orbit reduces to a fundamental orbit when $\sigma_{1}=\sigma_{2}$.

TheOREM 3.3: The $\sigma_{1} \sigma_{2}$-ORBITs. Let $\left(\alpha_{1}, \alpha_{2}, \beta_{1}, \beta_{2}, \mu\right)$ satisfy (1.2). Choose $\sigma_{1}, \sigma_{2} \in\left\{A_{N}, B_{M}\right\}_{N, M \geq 1}$ for some $N$ and $M$ such that $\sigma_{1} \neq \sigma_{2}$. Then, for any $0<C<C_{\max (N, M)}^{\mathrm{SN}}$ and $\varepsilon>0$ small enough, there exists a periodic orbit $\gamma_{\sigma_{1} \sigma_{2}}^{\mathrm{per}}(\xi ; C)$ with positive $u, v$ coordinates such that $\gamma_{\sigma_{1} \sigma_{2}}^{\text {per }}(\xi ; C)$ consists of two fast jumps away from and back to $\mathcal{M}$ and two slow segments near $\mathcal{M}$. Of the fast jumps, one is of $\sigma_{1-}$ type and the other of $\sigma_{2}$-type; and, of the slow segments, one is exponentially close to that part of $\Gamma_{C} \subset \mathcal{M}$ in between $P_{\sigma_{1}}^{-}(C)$ and $P_{\sigma_{2}}^{+}(C)$, while the other is exponentially close to that part of $\Gamma_{C}$ in between $P_{\sigma_{2}}^{-}(C)$ and $P_{\sigma_{1}}^{+}(C)$.

We refer to Figure $1.1 \mathrm{~b}$ for (a part) of the $A_{1} B_{1}$-orbit as solution of the PDE (1.4) and to Figure 3.2 for a sketch of the same orbit in the 4-dimensional phase space associated to (1.6).

Proof of Theorem 3.3. Choose $\sigma_{1,2} \in\left\{A_{N}, B_{M}\right\}_{N, M \geq 1}$, with $\sigma_{1} \neq \sigma_{2}$, and $C$ such that $0<C<C_{\max (N, M)}^{\mathrm{SN}}$, where $N$ is determined by $\sigma_{1}$ and $M$ by $\sigma_{2}$. Consider the fundamental periodic orbit $\gamma_{\sigma_{1}}^{\text {per }}(\xi)=\left(u_{\sigma_{1}}(\xi), p_{\sigma_{1}}(\xi), v_{\sigma_{1}}(\xi), q_{\sigma_{1}}(\xi)\right)$ (Theorem 3.1). By a translation in $\xi$ we can assume that $\gamma_{\sigma_{1}}^{\text {per }}(\xi)$ has its $N$-th intersection with $\{q=0\}$ at $\xi=0$, exponentially close to $\mathcal{I}_{+N}$. It follows from the construction of $\gamma_{\sigma_{1}}^{\text {per }}(\xi)$ (and the symmetry $\left.(1.7)\right)$ that $\gamma_{\sigma_{1}}^{\text {per }}(0)=\left(u_{\sigma_{1}}^{\text {extr }}, 0, v_{\sigma_{1}}^{\text {extr }}, 0\right)$ so that $u_{\sigma_{1}}^{\text {extr }}$ and $u_{\sigma_{1}}^{\text {extr }}$ are local extrema of $u_{\sigma_{1}}(\xi)$ and $v_{\sigma_{1}}(\xi)$. Note that $u_{\sigma_{1}}^{\text {extr }}$ and $v_{\sigma_{1}}^{\text {extr }}$ are absolute maxima when $N=1$ and local minima for $N$ even (see Figures 1.1a, 3.1a and 3.1b).

As in the proof of Theorem 3.1 we consider the two-dimensional manifold of orbits through an exponentially small one-dimensional interval, $I_{\sigma_{1}} \subset\left\{p=q=0, u=u_{\sigma_{1}}^{\text {extr }}\right\}$ 


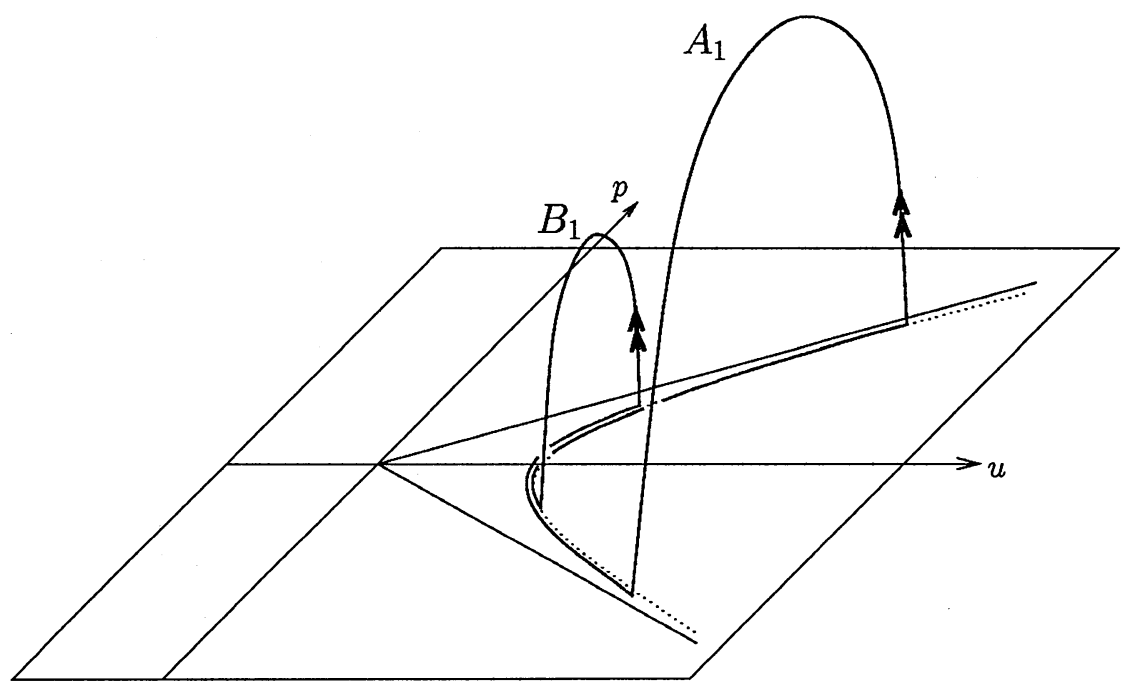

FIG. 3.2. A schematic illustration of an orbit of 'mixed' type, $\gamma_{A_{1} B_{1}}^{\mathrm{per}}(\xi ; C)$.

with $\gamma_{\sigma_{1}}^{\text {per }}(0) \in I_{\sigma_{1}}$. We know from the construction of $\gamma_{\sigma_{1}}^{\text {per }}(\xi)$ that it is $\mathcal{O}\left(e^{-1 / \varepsilon^{2}}\right)$-close to $W^{s}(\mathcal{M})$, and 'inside' $W^{s}(\mathcal{M})$ (the $v$-coordinate of an orbit that is 'outside' $W^{s}(\mathcal{M})$ will become negative). We choose one endpoint, $Q_{\sigma_{1}}^{s}$, of $I_{\sigma_{1}}$ to be at $W^{s}(\mathcal{M}) \cap\{p=$ $\left.q=0, u=u_{\sigma_{1}}^{\text {extr }}\right\}$, i.e., $Q_{\sigma_{1}}^{s} \in \mathcal{I}_{-N}$. Note that $Q_{\sigma_{1}}^{s}$ has a $v$-coordinate that is larger than $v_{\sigma_{1}}^{\text {extr }}$ when $N$ is odd, and smaller than $v_{\sigma_{1}}^{\text {extr }}$ when $N$ is even. The other endpoint, $Q_{\sigma_{1}}^{o}$, of $I_{\sigma_{1}}$ is assumed to be situated at the other side of $\gamma_{\sigma_{1}}^{\text {per }}(0)$.

Now consider an orbit $\gamma(\xi)=\left(u(\xi), p(\xi), v(\xi), q(\xi)\right.$ with $\gamma(0) \in I_{\sigma_{1}}$. This orbit will follow $\gamma_{\sigma_{1}}^{\text {per }}(\xi)$ for $\frac{N}{2}$ circuits, or $N$ 'half-circuits', through the fast field and will touch down on $\mathcal{M}$ exponentially close to $P_{\sigma_{1}}^{-}(C) \in \Gamma_{C}(2.37)$. It is asymptotic to $\mathcal{M}$ when $\gamma(0)=Q_{\sigma_{1}}^{s}$, it will take off from $\mathcal{M}$ at $P_{\sigma_{1}}^{+}(C)$ when $\gamma(0)=\gamma_{\sigma_{1}}^{\text {per }}(0)$. If $\gamma(0)$ is too far away from $W^{s}(\mathcal{M}), \gamma(\xi)$ will again take off from $\mathcal{M}$ 'immediately' at the touch down point $P_{\sigma_{1}}^{-}(C)$. Hence, it follows that one can choose $v(0)=v_{\ell}$, respectively $v(0)=v_{u}$, such that $\gamma(\xi)$ touches down on $\mathcal{M}$ near $P_{\sigma_{1}}^{-}(C)$, follows $\Gamma_{C}$ and takes off again before, respectively after, it reaches $P_{\sigma_{2}}^{+}(C)$, the take off point associated to the $\sigma_{2}$-jump.

We can now use similar arguments as in the proof of Theorem 3.1. The manifold $\mathcal{L}_{\sigma_{1}}$ spanned by orbits with initial conditions on $I_{\sigma_{1}}$ with $v$-coordinates between $v_{\ell}$ and $v_{u}$ is, of course, exponentially close to $W^{u}\left(\Gamma_{C}\right)$. The $M$-th intersection of $\mathcal{L}_{\sigma_{1}}$ with $\{q=0\}$ is exponentially close to the $M$-th intersection of $W^{u}\left(\Gamma_{C}\right)$ with $\{q=0\}$, i.e. $W^{u}\left(\Gamma_{C}\right) \cap \mathcal{I}_{+M}$. The curve of the $M$-th branch of $\mathcal{L}_{\sigma_{1}} \cap\{q=0\}$ crosses the plane $\{p=0\}$ (see the proof of Theorem 3.1), which implies that there must be a point in the $M$-th intersection of $\mathcal{L}_{\sigma_{1}}$ with $\{q=0\}$ with $p$-coordinate $=0$.

Hence, we have constructed an orbit $\gamma_{\sigma_{1} \sigma_{2}}^{\text {per }}(\xi)$ with initial condition on $I_{\sigma_{1}} \subset\{p=$ $q=0$ \} that makes $\frac{N}{2}$ circuits through the fast field before it touches down on $\mathcal{M}$ near $P_{\sigma_{1}}^{-}(C)$. On $\mathcal{M}$, it follows $\Gamma_{C}$ until it takes off near $P_{\sigma_{2}}^{+}(C)$ and makes $\frac{M}{2}$ circuits through the fast field, after which it again intersects $\{p=q=0\}$. By the symmetry $(1.7)$, we can 'double' this 'half-orbit' into a 'full' periodic orbit $\gamma_{\sigma_{1} \sigma_{2}}^{\text {per }}(\xi)$ of $\sigma_{1} \sigma_{2}$-type. 口

The construction of the $\gamma_{\sigma_{1} \sigma_{2}}^{\mathrm{per}}(\xi)$ orbits is clearly based on the existence of the 'fundamental' $\gamma_{\sigma_{1}}^{\text {per }}(\xi)$ orbits. By extending the procedure used in the above proof, we 
can construct more than just the $\sigma_{1} \sigma_{2}$-orbits from the fundamental orbits.

For instance, it is a straightforward procedure to construct $\sigma_{1}^{2 n+1} \sigma_{2}$-orbits $(n \geq 1)$ by the methods of the proof of Theorem 3.3. The idea is to again take an interval of initial conditions $\subset\{p=q=0\}$ exponentially close to $\mathcal{I}_{-N}$ and to $\gamma_{\sigma_{1}}^{\text {per }}(0)$, that is translated to be $\subset\{p=q=0\}$ 'halfway' along the $\sigma_{1}$-jump of $\gamma_{\sigma_{1}}^{\text {per }}(\xi)$, and to consider an orbit $\gamma^{\text {per }}(\xi)$ that follows $\gamma_{\sigma_{1}}^{\text {per }}(\xi)$ for a longer time than $\gamma_{\sigma_{1} \sigma_{2}}^{\text {per }}(\xi)$. This orbit $\gamma^{\text {per }}(\xi)$ also remains exponentially close to $\gamma_{\sigma_{1}}^{\text {per }}(\xi)$ during the next $\sigma_{1}$-jump of $\gamma_{\sigma_{1}}^{\text {per }}(\xi)$ through the fast field until $\gamma_{\sigma_{1}}^{\text {per }}(\xi)$ touches down again on $\mathcal{M}$. After this full $\sigma_{1}$-jump we let $\gamma^{\text {per }}(\xi)$ take off at $P_{\sigma_{2}}^{+}(C)$ in such a way that $\gamma^{\text {per }}(\xi)$ crosses $\{p=q=0\}$ after $\frac{M}{2}$ circuits of $\sigma_{2}$-type (exponentially close to $\mathcal{I}_{+M}$ ). Thus, half of $\gamma^{\text {per }}(\xi)$ consists of half a $\sigma_{1}$-jump, followed (after a slow piece on $\mathcal{M}$ ) by a full $\sigma_{1}$-jump and half a $\sigma_{2^{-}}$ jump. We apply the symmetry (1.7) and conclude that $\gamma^{\text {per }}(\xi)$ is a periodic orbit that produces a solution of the PDE consisting of the sequence $\ldots \sigma_{1} \sigma_{1} \sigma_{1} \sigma_{2} \sigma_{1} \sigma_{1} \sigma_{1} \sigma_{2} \sigma_{1} \ldots$ of pulses/jumps, namely $\gamma^{\mathrm{per}}(\xi)=\gamma_{\sigma_{1}^{3} \sigma_{2}}^{\mathrm{per}}(\xi)$. This is a periodic orbit of $\sigma_{1}^{3} \sigma_{2}$-type. For each $n \geq 1$, the $\sigma_{1}^{2 n+1} \sigma_{2}$-orbit can be constructed in a similar fashion.

Moreover, we can use the existence of the $\sigma_{1} \sigma_{2}$-orbits to construct orbits that make jumps of 3 different types, $\sigma_{1}, \sigma_{2}$ and $\sigma_{3}$ (with $\sigma_{1,2,3} \in\left\{A_{N}, B_{M}\right\}_{N, M \geq 1}$ ). Again we can follow the construction in the proof of Theorem 3.3. We construct an orbit $\gamma^{\text {per }}(\xi)$ that has its initial condition in $\{p=q=0\}$, exponentially close to $\mathcal{I}_{-N}$ and to $\gamma_{\sigma_{1} \sigma_{2}}^{\text {per }}(\xi)$, 'halfway' along the $\sigma_{1}$-jump; $\gamma^{\text {per }}(\xi)$ follows $\gamma_{\sigma_{1} \sigma_{2}}^{\text {per }}(\xi)$ along the first 'half' $\sigma_{1}$-jump, along $\mathcal{M}$, and along the full $\sigma_{2}$-jump back to $\mathcal{M}$. Then, we can make $\gamma^{\text {per }}(\xi)$-jump at $P_{\sigma_{3}}^{+}(C)$ and let it make half a $\sigma_{3}$-jump (of $N_{3} / 2$ circuits, where $N_{3}$ is determined by $\left.\sigma_{3}\right)$ to arrive, once again, at $\{p=q=0\}$, exponentially close to $\mathcal{I}_{+N_{3}}$. Note that we have to assume that $C$ is small enough, i.e. that $\Gamma_{C}$ has a non-empty intersection with the take off set $T_{N_{3}}^{\text {off }}$ associated to the $\sigma_{3}$-jump. It follows that half of $\gamma^{\text {per }}(\xi)$ consists of half a $\sigma_{1}$-jump, followed by a full $\sigma_{2}$-jump and half a $\sigma_{3}$-jump: $\gamma^{\text {per }}(\xi)$ is associated to a pattern with a $\ldots \sigma_{1} \sigma_{2} \sigma_{3} \sigma_{2} \sigma_{1} \sigma_{2} \sigma_{3} \sigma_{2} \sigma_{1} \sigma_{2} \ldots$ sequence of singular pulses, $\gamma^{\text {per }}(\xi)=\gamma_{\sigma_{1} \sigma_{2} \sigma_{3} \sigma_{2}}^{\text {per }}(\xi)$, a $\sigma_{1} \sigma_{2} \sigma_{3} \sigma_{2}$-orbit.

Every family of periodic orbits we have so far constructed can again be used as foundation for the construction of a next, more complicated, family of orbits. And so on. The main question we need to answer is: What kind of multi-pulse periodic patterns can, and what kind cannot, be constructed?

Of course, the use of the reversibility symmetry (1.7) is crucial for the construction of the periodic orbits. Every orbit is constructed by establishing that there is a connection possible from $\{p=q=0\}$ back to $\{p=q=0\}$. This connection is 'half an orbit', and the symmetry (1.7) makes it into a full, closed periodic orbit. This implies that any periodic orbit constructed by this procedure has (at least) two internal reflection symmetries, one at $\xi=0$ (after translation), $\gamma^{\mathrm{per}}(-\xi)=\gamma^{\mathrm{per}}(\xi)$, and one halfway along the period $T$ of the orbit, $\gamma^{\text {per }}\left(\frac{T}{2}-\xi\right)=\gamma^{\text {per }}\left(\frac{T}{2}+\xi\right)$.

A periodic orbit cannot have only one internal reflection symmetry, since the existence of the second symmetry can be deduced from the first symmetry by application of the periodicity of $\gamma^{\text {per }}(\xi)$ :

$$
\gamma^{\mathrm{per}}\left(\frac{T}{2}-\xi\right)=\gamma^{\mathrm{per}}\left(-\frac{T}{2}+\xi\right)=\gamma^{\mathrm{per}}\left(\frac{T}{2}+\xi\right)
$$

Moreover, a periodic orbit with more than two internal reflection symmetries must correspond to a periodic orbit with less symmetries and a shorter period: assume that $\gamma^{\text {per }}(\tilde{T}-\xi)=\gamma^{\text {per }}(\tilde{T}+\xi)$ for some $\tilde{T} \leq \frac{T}{2}$, then

$$
\gamma^{\text {per }}(2 \tilde{T}+\xi)=\gamma^{\text {per }}(-\xi)=\gamma^{\text {per }}(\xi)
$$


i.e. $\gamma^{\text {per }}(\xi)$ is $2 \tilde{T}$-periodic. Thus, any periodic orbit $\gamma^{\text {per }}(\xi)$ either has exactly two internal reflection symmetries (around 0 and $\frac{T}{2}$ ) or no symmetries.

It follows from simple geometric counting arguments that one cannot expect that there exist one-parameter families of periodic orbits without an internal reflection symmetry, since one cannot use the reversibility symmetry (1.7) to construct these orbits. We do not investigate the possible existence of these 'degenerate periodic orbits' in any further detail.

Finally, we show that, for every possible periodic symbol sequence with two internal reflection symmetries, there exists a one-parameter family of singular periodic orbits that has precisely that sequence of pulses:

THEOREM 3.4: THE $\tau \tau^{-1}-, \tau \sigma \tau^{-1}$ - AND $\sigma \tau \tilde{\sigma} \tau^{-1}$-ORBITS. Let $\left(\alpha_{1}, \alpha_{2}, \beta_{1}, \beta_{2}, \mu\right)$ satisfy (1.2). Let $\sigma, \tilde{\sigma}, \sigma_{j} \in\left\{A_{N}, B_{M}\right\}_{N, M \geq 1}, j=1,2,3, \ldots$; let $\tau=\sigma_{1} \sigma_{2} \ldots \sigma_{J}$ be a random sequence of $J \sigma_{j}$ 's, and define $\tau^{-1}$ by $\tau^{-1}=\sigma_{J} \sigma_{J-1} \ldots \sigma_{1}$; let $\varepsilon>0$ be small enough. Then there exists one-parameter families of periodic solutions of (1.6) of $\tau \tau^{-1}$-type, of $\tau \sigma \tau^{-1}$-type, and of $\sigma \tau \tilde{\sigma} \tau^{-1}$-type. Moreover, any possible periodic orbit with internal reflection symmetries is of one of these types.

We refer back to Figure 1.2 for a sketch of an orbit $\gamma_{A_{1} A_{2} A_{3} A_{2} A_{1}}^{\text {per }}(\xi)$ as solution of the PDE. This is an orbit of $\tau \sigma \tau^{-1}$-type, with $\tau=A_{1} A_{2}$ and $\sigma=A_{3}$. In terms of the notation of Theorem 3.4, the $\sigma_{1} \sigma_{2}$-orbits described in Theorem 3.3 are of $\sigma \tau \tilde{\sigma} \tau^{-1}$-type with $\sigma=\sigma_{1}, \tau=\emptyset$ and $\tilde{\sigma}=\sigma_{2}$. Also, the fundamental periodic solutions of Theorem 3.1 are of $\tau \sigma \tau^{-1}$-type with, again, $\tau=\emptyset$. In section 4 , a very natural interpretation is presented of the three different types of periodic orbits as multi-pulse patterns of the PDE on a bounded domain with homogeneous Neumann boundary conditions.

Proof of Theorem 3.4. A symmetric periodic orbit $\gamma^{\text {per }}(\xi)$ must have its reflection symmetry points, i.e., its intersections with $\{p=q=0\}$, either exponentially close to $\mathcal{M}$ or in the fast field, halfway along a jump of $\sigma$-type for a certain $\sigma$, i.e., exponentially close to an $N$-th intersection $\mathcal{I}_{ \pm N}$ of $W^{u, s}(\mathcal{M})$ and $\{q=0\}$, where $N$ is determined by $\sigma$. There are three different possible connections from $\{p=q=0\}$ to $\{p=q=0\}$.

In the first one, $\gamma^{\mathrm{per}}(\xi)$ is exponentially $\left(\mathcal{O}\left(e^{-1 / \varepsilon^{2}}\right)\right)$ close to $\mathcal{M}$ both at $\xi=0$ and at $\xi=\frac{T}{2}$. The second one is encountered in the proof of Theorem 3.1: at $\xi=0$, $\gamma^{\text {per }}(\xi)$ is exponentially close to $\mathcal{M}$, while at $\xi=\frac{T}{2}, \gamma^{\text {per }}(\xi)$ is exponentially close to an $\mathcal{I}_{N}$ in the fast field. The third one appears in the proof of Theorem 3.3: at $\xi=0$, $\gamma^{\text {per }}(\xi)$ is exponentially close to an $\mathcal{I}_{-N}$, and at $\xi=\frac{T}{2}, \gamma^{\text {per }}(\xi)$ is again exponentially close to an $\mathcal{I}_{+\tilde{N}}$.

We first consider the third case. Here, we have to put $\gamma^{\text {per }}(0) \in\{p=q=0\}$ halfway along a $\sigma$-jump, exponentially close to $\mathcal{I}_{-N} ; \gamma^{\text {per }}\left(\frac{T}{2}\right) \in\{p=q=0\}$ is halfway along a $\tilde{\sigma}$-jump, exponentially close to $\mathcal{I}_{+\tilde{N}}$. The behavior of $\gamma^{\text {per }}(\xi)$ in between the $\sigma$ - and $\tilde{\sigma}$-jumps is prescribed by the 'random sequence' $\tau$. Hence, by the symmetry, these orbits are of $\sigma \tau \tilde{\sigma} \tau^{-1}$-type.

The first nontrivial example has already been constructed above, just after the proof of Theorem 3.3: $\gamma_{\sigma_{1} \sigma_{2} \sigma_{3} \sigma_{2}}^{\text {per }}(\xi)$ is an orbit of $\sigma \tau \tilde{\sigma} \tau^{-1}$-type, with $\sigma=\sigma_{1}, \tau=\sigma_{2}$ and $\tilde{\sigma}=\sigma_{3}$. This orbit is constructed from $\gamma_{\sigma_{1} \sigma_{2}}^{\text {per }}(\xi)$ in the same manner as $\gamma_{\sigma_{1} \sigma_{2}}^{\text {per }}(\xi)$ has been constructed from $\gamma_{\sigma_{1}}^{\text {per }}(\xi)$ in the proof of Theorem 3.3. Furthermore, by exactly the same methods, it is possible to use $\gamma_{\sigma_{1} \sigma_{2} \sigma_{3} \sigma_{2}}^{\mathrm{per}}(\xi)$ as the foundation for the construction of the orbit $\gamma_{\sigma_{1} \sigma_{2} \sigma_{3} \sigma_{4} \sigma_{3} \sigma_{2}}^{\text {per }}(\xi)$, an orbit of $\sigma \tau \tilde{\sigma} \tau^{-1}$-type with $\sigma=\sigma_{1}$, $\tau=\sigma_{2} \sigma_{3}$ and $\tilde{\sigma}=\sigma_{4}$. Therefore, any $\sigma \tau \tilde{\sigma} \tau^{-1}$-orbit, with $\tau$ of finite length, can be constructed by the iteration of this procedure.

The second case corresponds to the periodic orbits of $\tau \sigma \tau^{-1}$-type. As mentioned 
above, these orbits can be constructed by the iteration of the procedure described in the proof of Theorem 3.1. The first step is the construction of the $\tau \sigma \tau^{-1}$-orbit $\gamma_{\sigma_{1} \sigma_{2} \sigma_{1}}^{\text {per }}(\xi)$ (i.e. $\tau=\sigma_{1}, \sigma=\sigma_{2}$ ), which follows by combining the ideas of the proof of Theorem 3.1 with the construction of the $\sigma_{1} \sigma_{2} \sigma_{3} \sigma_{2}$-orbit above. In particular, we place $\gamma_{\sigma_{1} \sigma_{2} \sigma_{1}}^{\text {per }}(0)$ in the exponentially small interval $I_{A, B} \in\{p=q=0\}$ exponentially close to $\mathcal{M} ; I_{A, B}$ was already defined in the proof of Theorem 3.1. The orbit $\gamma_{\sigma_{1} \sigma_{2} \sigma_{1}}^{\text {per }}(\xi)$ follows $\gamma_{\sigma_{1}}^{\text {per }}(\xi)$ along $\Gamma_{C}$ and during the $\sigma_{1}$-jump through the fast field. After $\gamma_{\sigma_{1} \sigma_{2} \sigma_{1}}^{\text {per }}(\xi)$ has touched down again on $\mathcal{M}$, it follows $\Gamma_{C}$, but eventually diverges from $\gamma_{\sigma_{1}}^{\text {per }}(\xi)$. We tune the initial condition on $I_{A, B}$ such that $\gamma_{\sigma_{1} \sigma_{2} \sigma_{1}}^{\text {per }}(\xi)$ takes off near $P_{\sigma_{2}}^{+}$, and so that it makes its $N_{2}$-th intersection at $\xi=\frac{T}{2}$ with $\{p=q=0\}$, exponentially close to $\mathcal{I}_{+N_{2}}$. Thus, $\gamma_{\sigma_{1} \sigma_{2} \sigma_{1}}^{\text {per }}(\xi)$ is exactly an orbit of $\sigma_{1} \sigma_{2} \sigma_{1}$-type. Next, we use $\gamma_{\sigma_{1} \sigma_{2} \sigma_{1}}^{\text {per }}(\xi)$ to construct $\gamma_{\sigma_{1} \sigma_{2} \sigma_{3} \sigma_{2} \sigma_{1}}^{\text {per }}(\xi)$, the next $\tau \sigma \tau^{-1}$-orbit with $\tau=\sigma_{1} \sigma_{2}$ and $\sigma=\sigma_{3}$. And so on.

So far, we have not yet constructed an orbit of $\tau \tau^{-1}$-type, described above as case one. The first nontrivial example is $\gamma_{\sigma_{1} \sigma_{2} \sigma_{2} \sigma_{1}}^{\text {per }}(\xi)$, where $\tau=\sigma_{1} \sigma_{2}$. This orbit can be constructed from the fundamental orbit $\gamma_{\sigma_{1}}^{\text {per }}(\xi)$. We again consider the interval of initial conditions $I_{A, B} \in\{p=q=0\}$, and again consider a family $\mathcal{L}_{A, B}$ of orbits with initial conditions in $I_{A, B}$ (see the proof of Theorem 3.1) that follow $\gamma_{\sigma_{1}}^{\text {per }}(\xi)$ along $\Gamma_{C}$, and during its first $\sigma_{1}$-jump through the fast field. By tuning the initial conditions, we can make any orbit $\gamma(\xi) \in \mathcal{L}_{A, B}$ take off near $P_{\sigma_{2}}^{+}$and have it make a jump of $\sigma_{2}$-type through the fast field. After touch down, $\gamma(\xi)$ can be made to intersect $\{p=0\}$ again, since $\gamma(\xi)$ follows the slow flow on $\Gamma_{C}$ and any $\Gamma_{C}$ intersects $\{p=0\}$ on $\mathcal{M}$ (Figure 2.1).

In order to apply the symmetry (1.7), we need to show that there is a special orbit $\gamma_{\sigma_{1} \sigma_{2} \sigma_{2} \sigma_{1}}^{\text {per }}(\xi) \in \mathcal{L}_{A, B}$ that first makes the $\sigma_{1^{-}}$and the $\sigma_{2}$-jumps, touches down on $\mathcal{M}$ and has its $q$-coordinate identically 0 at its next intersection with $\{p=0\}$.

The existence of this orbit follows by studying (1.6) near $\mathcal{M}$ in its Fenichel normal form $[18,19,26]$. Here, we refrain from presenting all computational details and only sketch the main ideas. Since the eigenvalues of the fast reduced limit problem (2.1) do not depend on $u^{0}$, we conclude that the linearized fast flow near $\mathcal{M}$ is, to leading order, not influenced by the values of $u$ and $p$. As a consequence, it follows from the normal form analysis that an orbit $\gamma(\xi)$ that touches down on $\mathcal{M}$ near a point $P^{-}$at $\xi=\Xi^{\text {down }}$ (approximately) and takes off again at $\xi=\Xi^{\text {off }}$ after an $\mathcal{O}\left(1 / \varepsilon^{2}\right)$ amount of time near a point $P^{+}$, has a minimal $v$-coordinate of $\mathcal{O}\left(e^{-1 / \varepsilon^{2}}\right)$ at a point that is halfway between $P^{-}$and $P^{+}$(to leading order), i.e. at $\xi^{\text {min }}=\frac{1}{2}\left(\Xi^{\text {off }}+\Xi^{\text {down }}\right)+$ h.o.t. Thus, $\gamma(\xi)$ intersects $\{q=0\}$ at $\xi^{\mathrm{min}}$, by definition $((1.6): q=\dot{v})$.

The application of the Fenichel normal form analysis to the construction of $\gamma_{\sigma_{1} \sigma_{2} \sigma_{2} \sigma_{1}}^{\text {per }}(\xi)$ yields that an orbit $\gamma(\xi) \in \mathcal{L}_{A, B}$, which touches down on $\mathcal{M}$ near $P_{\sigma_{2}}^{-}$and jumps off from $\mathcal{M}$ just after it has passed $\{p=0\}$, will reach a minimal (exponentially small) distance to $\mathcal{M}$ halfway between $P_{\sigma_{2}}^{-}$and $\{p=0\}$, i.e. $q=0$ with $p<0$ (Figure 2.1). An orbit $\gamma(\xi)$ that follows $\Gamma_{C}$ beyond $P_{\sigma_{2}}^{+}$, the take off point of the $\sigma_{2}$-jump, spends more time near the $\{p>0\}$-part of $\mathcal{M}$ that near the $\{p<0\}$-halfplane, hence it intersects $\{q=0\}$ with a positive $p$-coordinate. It follows that there is a uniquely determined orbit $\gamma(\xi)=\gamma_{\sigma_{1} \sigma_{2} \sigma_{2} \sigma_{1}}^{\mathrm{per}}(\xi) \in \mathcal{L}_{A, B}$ that intersects $\{p=q=0\}$ exponentially close to $\mathcal{M}$, after a full $\sigma_{1}$-jump and a full $\sigma_{2}$-jump (one need the details of the normal form analysis to conclude that $\gamma_{\sigma_{1} \sigma_{2} \sigma_{2} \sigma_{1}}^{\mathrm{per}}(\xi)$ is uniquely determined). Note that the normal form arguments also imply that $\gamma_{\sigma_{1} \sigma_{2} \sigma_{2} \sigma_{1}}^{\text {per }}(\xi)$ takes off from $\mathcal{M}$ (after the $\sigma_{1,2}$-jumps) near $P_{\sigma_{2}}^{+}$. This agrees with the final construction of $\gamma_{\sigma_{1} \sigma_{2} \sigma_{2} \sigma_{1}}^{\text {per }}(\xi)$ by the application of the symmetry (1.7). 
Now, as in the second and third case above, it is a straightforward procedure to construct the next $\tau \tau^{-1}$-orbit $\gamma_{\sigma_{1} \sigma_{2} \sigma_{3} \sigma_{3} \sigma_{2} \sigma_{1}}^{\text {per }}(\xi)$ by first following $\gamma_{\sigma_{1} \sigma_{2} \sigma_{2} \sigma_{1}}^{\text {per }}(\xi)$ for two full jumps. Hence, we can also construct all periodic orbits of $\tau \tau^{-1}$-type by an iterative process: the orbit with $\tau=\sigma_{1} \sigma_{2} \ldots \sigma_{k} \sigma_{k+1}$ is based on its predecessor with $\tau=\sigma_{1} \sigma_{2} \ldots \sigma_{k}$

This completes the proof of the theorem.

REMARK 3.5. One could a priori think that an orbit $\gamma^{\text {per }}(\xi)$ could be both of $\sigma \tau \tilde{\sigma} \tau^{-1}$-type and of $\tau \tau^{-1}$-type (these orbits have an even number of jumps through the fast field, whereas the $\tau \sigma \tau^{-1}$-orbits make an odd number of jumps). However, such orbits would have more than two internal reflection symmetries, and it follows from the arguments above (3.2) that one should be able to reduce these orbits to orbits with a shorter period. A direct check reveals: if $\gamma^{\mathrm{per}}(\xi)$ is both of $\tau \tau^{-1}$ - and of $\sigma \tau \tilde{\sigma} \tau^{-1}$-type, then $\tilde{\sigma} \tau^{-1}$ should be equal to $(\sigma \tau)^{-1}$, i.e. with $\tau=\sigma_{1} \sigma_{2} \ldots \sigma_{J}$ :

$$
\tilde{\sigma}=\sigma_{J}=\sigma, \sigma_{1}=\sigma_{J-1}, \sigma_{2}=\sigma_{J-2}, \text { etc. }
$$

Hence, if $J$ is odd, $\sigma \tau \tilde{\sigma} \tau^{-1}=\sigma \tau(\sigma \tau)^{-1}=\tilde{\tau} \tilde{\tau}^{-1} \tilde{\tau} \tilde{\tau}^{-1}$, with $\tilde{\tau}=\sigma \sigma_{1} \ldots \sigma_{(J-1) / 2}$. The orbit $\gamma^{\text {per }}(\xi)$ with a periodic $\sigma$-jump sequence of $2 J+2$ elements is in fact a $\tau \tau^{-1}$ orbit with a periodic sequence of half the number of pulses, $J+1$. If $J$ is even, $\sigma \tau \tilde{\sigma} \tau^{-1}=\left(\hat{\tau} \hat{\sigma} \hat{\tau}^{-1}\right)^{2}$ with $\hat{\tau}=\sigma \sigma_{1} \ldots \sigma_{J / 2-1}, \hat{\sigma}=\sigma_{J / 2}$, which implies that $\gamma^{\text {per }}(\xi)$ is in fact an orbit of $\tau \sigma \tau^{-1}$-type, again with a periodic sequence of $J+1$ elements.

4. Random, singular, spatial patterns on sufficiently large bounded intervals. It is natural to consider solutions of the PDE (1.4) on a bounded interval $(0, L)$ with homogeneous Neumann boundary conditions (or with other boundary conditions, see also Remark 4.2). In the terminology of the dynamical system (1.6), these boundary conditions correspond to $p=q=0$ at $\xi=0$ and $\xi=L / \varepsilon$. Note that this implies that we have to consider $L=\tilde{L} / \varepsilon$, since the distance (as measured in $\xi$ ) between successive pulses of a multi-pulse pattern of $A_{1}, B_{1}$-type is of $\mathcal{O}\left(1 / \varepsilon^{2}\right)$. Thus, we can take $L / \varepsilon$, or $\tilde{L} / \varepsilon^{2}$, to be $\frac{T}{2}$, i.e. half the period of a periodic solution $\gamma^{\text {per }}(\xi)$.

The three different types of orbits described in Theorem 3.4 have a very natural interpretation in this context, since they can also be distinguished by their character near $\xi=0$ and $\xi=\frac{T}{2}$ (section 3.2). An orbit of $\tau \tau^{-1}$-type corresponds to a multipulse pattern $\left(U_{\tau \tau^{-1}}^{\text {per }}(x), V_{\tau \tau^{-1}}^{\text {per }}(x)\right)$ that has no pulses at the boundaries; $V_{\tau \tau^{-1}}^{\text {per }}(x)$ is exponentially small at $\xi=0$ and $\xi=\frac{T}{2}$ since $\gamma_{\tau \tau^{-1}}^{\text {per }}(0)$ and $\gamma_{\tau \tau^{-1}}^{\text {per }}\left(\frac{T}{2}\right)$ are both exponentially close to $\mathcal{M}$. Moreover, both $U_{\tau \tau^{-1}}^{\text {per }}(x)$ and $V_{\tau \tau^{-1}}^{\text {per }}(x)$ are at a local minimum at the boundaries. The pattern $\left(U_{\tau \sigma \tau^{-1}}^{\text {per }}(x), V_{\tau \sigma \tau^{-1}}^{\text {per }}(x)\right)$, that corresponds to a $\tau \sigma \tau^{-1}$-orbit, has a similar structure near one of the boundaries, say at $\xi=0$, however, $\gamma_{\tau \sigma \tau^{-1}}^{\text {per }}(\xi)$ is halfway along a pulse of $\sigma$-type at the other boundary, $\xi=\frac{T}{2}$. Thus, $V_{\tau \sigma \tau^{-1}}^{\text {per }}(x)$ generates half a pulse of $\sigma$-type, i.e. $\frac{N}{2}$ pulses (where $N$ is determined by $\sigma$ ), at this boundary. The $U$-component $U_{\tau \sigma \tau^{-1}}^{\text {per }}(x)$ is at a local minimum at the $\xi=0$ boundary and exhibits half a ('slow') $U$-pulse at the $\xi=\frac{T}{2}$ boundary. The $\left(U_{\sigma \tau \tilde{\sigma} \tau^{-1}}^{\text {per }}(x), V_{\sigma \tau \tilde{\sigma} \tau^{-1}}^{\text {per }}(x)\right)$ patterns have 'half-pulses' at both boundaries, one of $\sigma$-type at $\xi=0$ and one of $\tilde{\sigma}$-type at $\xi=\frac{T}{2}$. See Figures 1.1a and 1.1b and Remark 4.3.

Now, we can reformulate Theorem 3.4 in terms of a result on random, in general aperiodic, patterns on bounded intervals:

CoRollary 4.1: 'RANDOM' SINGULAR PATTERNS ON BOUNDED INTERVALS. Let $\left(\alpha_{1}, \alpha_{2}, \beta_{1}, \beta_{2}, \mu\right)$ satisfy (1.2). Let $\tilde{\tau}$ be a random sequence of $\tilde{J} \sigma$ 's, $\left(\sigma_{j} \in\right.$ $\left.\left\{A_{N}, B_{M}\right\}_{N, M \geq 1}, j=1,2,3, \ldots, \tilde{J}\right)$, with $\sigma_{1}$ and $\sigma_{\tilde{J}}$ such that they either correspond 
to a full pulse of $\sigma_{1}$ or $\sigma_{\tilde{J}}$-type or to half a pulse of $\sigma_{1}$-or $\sigma_{\tilde{J}}$-type at $\xi=0$ or $\xi=L$; let $\varepsilon>0$ be small enough. Then there exists an $L_{\min }>0$ such that for all $L>L_{\min }$ the PDE (1.4) defined on the interval $(0, L)$ with homogeneous Neumann boundary conditions has a 'random' multi-pulse pattern of the type described by the sequence $\tilde{\tau}$.

Note that the fact that there exist 'one-parameter families' in Theorem 3.4 has been translated into the existence of a continuum of allowed intervals $(0, L)$; the limit $L \rightarrow \infty$ corresponds to considering orbits $\Gamma_{C}$ on $\mathcal{M}$ with $C \downarrow 0$.

REMARK 4.2. The geometric singular perturbation approach of sections 2 and 3 can also be applied to the construction of singular multi-pulse patterns on bounded intervals with other types of boundary conditions, such as homogeneous Dirichlet conditions. We do not go into the details here.

REMARK 4.3. By choosing the length $L$ of the interval $(0, L)$ such that $L=k T$, for a fixed integer $k=1,2,3, \ldots$, i.e., by taking $L$ such that it is equal to an even multiple of $\frac{T}{2}$, a pattern of $\tau \sigma \tau^{-1}$-type can either have no pulses at the boundary or pulses at both boundaries (here, we of course assume that the solution satisfies homogeneous Neumann boundary conditions). Thus, for instance the fundamental solutions (where $\tau$ is the empty set) can be plotted with or without pulses at the boundaries of the domain. See Figure 1.1a, where $L=4 T=8 \frac{T}{2}$, and compare it to the fundamental periodic patterns in the Gray-Scott model in [4] that have been plotted without pulses on the boundaries. Solutions of $\tau \tau^{-1}$-type, respectively $\sigma \tau \tilde{\sigma} \tau^{-1}$-type, can only be represented on finite domains (again assuming homogeneous Neumann boundary conditions) by patterns without pulses at the boundary, respectively with pulses at both boundaries (Figure 1.1b). The most simple 'mixed' patterns of $\sigma_{1} \sigma_{2}$ type described in Theorem 3.3 are of $\sigma \tau \tilde{\sigma} \tau^{-1}$-type and thus cannot be plotted without pulses at both boundaries (Figure 1.1b). It follows that these solutions have not been considered in [29].

5. Discussion. We have shown in this paper that the generalized GiererMeinhardt equation (1.1)/(1.4) has an extremely rich set of distinct periodic and random solutions/patterns.

A very natural, and important, next question is: Can any of these patterns be stable? Let us focus our attention here on the spatially periodic patterns. It was shown in [5] that the homoclinic patterns $\left(U_{N}^{\text {hom }}(x), V_{N}^{\text {hom }}(x)\right)$ associated to the solutions $\gamma_{N}^{\text {hom }}(\xi)$ of (1.6) can only be stable for $N=1$ (and $\mu$ not too close to 0 ). When $N \geq 2$, the spectrum associated to the linearization of $(1.4)$ along $\left(U_{N}^{\text {hom }}(x), V_{N}^{\text {hom }}(x)\right)$ always has at least one unstable (real) eigenvalue [5]. The distance between successive $\sigma$-pulses in any periodic multi-pulse pattern is asymptotically large, therefore there will be a strong relation between the spectrum of the linearization along a periodic pattern and the spectra associated to the homoclinic $\gamma_{N}^{\text {hom }}(\xi)$ pulses (see [12] for a general treatment of this issue, and [4] for explicit computations of the spectra associated to singular periodic patterns in the Gray-Scott model). Hence, one cannot expect a periodic multi-pulse pattern that involves $\sigma$-pulses with $\sigma=A_{N}$ or $B_{M}$ and $N, M \geq 2$ to be stable as solution of the PDE. Of course this result has not yet been proved, but it provides a strong motivation to focus on the stability analysis of multi-pulse patterns that consist only of pulses of either $A_{1}$ - or $B_{1}$-type. Note that these are exactly the kind of patterns considered in [29] (see Remark 5.1).

Even when one restricts the kind of jumps through the fast field to the "potentially stable' types $A_{1}$ and $B_{1}$, the results of section 3 still produce a rich structure of spatially periodic patterns. It is, for instance, not immediately clear what 
is the minimum length of a sequence $\tilde{\tau}$ of $A_{1}$ 's and $B_{1}$ 's that is not of one of the symmetrical types described in Theorem 3.4. As an example, we consider the sequence $\tilde{\tau}=A_{1} A_{1} B_{1} B_{1} A_{1}$ of length $5 ; \tilde{\tau}$ corresponds to the periodic sequence of pulses $\ldots A_{1} A_{1} B_{1} B_{1} A_{1} A_{1} A_{1} B_{1} B_{1} A_{1} \ldots$, i.e. a pattern of $\tau \sigma \tau^{-1}$-type, with $\tau=B_{1} A_{1}$ and $\sigma=A_{1}$. An asymmetrical sequence $\tilde{\tau}$ must be at least of length 6 ; and, $\tilde{\tau}=A_{1} B_{1} B_{1} A_{1} A_{1} B_{1}$ is one of the first examples. Note however, that the fact that the sequence $\tilde{\tau}=A_{1} B_{1} B_{1} A_{1} A_{1} B_{1}$ is not symmetrical does not imply that it is not possible to have a solution with this structure, or a periodic iteration of it, on a bounded spatial interval (Corollary 4.1).

Finally, we briefly consider some of the subtleties associated to the distinction between having established the existence of symmetric periodic orbits in (1.6) in Theorem 3.4 and their interpretation as 'random', in general aperiodic (or asymmetric [29]), patterns on a bounded domain in Corollary 4.1. For instance, Corollary 4.1 establishes the existence of a pattern associated to the 'periodic' sequence of pulses $\tilde{\tau}=\left(A_{1} B_{1} B_{1} A_{1} A_{1} B_{1}\right)^{K}$ without pulses on the boundary, for any $K \geq 1$ (on intervals $(0, L)$ of sufficient length). Here, $A_{1} B_{1} B_{1} A_{1} A_{1} B_{1}$ is the above mentioned example of an asymmetric sequence of $A_{1}$ and $B_{1}$ pulses, hence, there is no one-parameter family of periodic orbits $\gamma_{A_{1} B_{1} B_{1} A_{1} A_{1} B_{1}}^{\text {per }}(\xi)$. Of course, the $\tilde{\tau}$-pattern on $(0, L)$ is associated to a symmetric periodic solution $\gamma_{(\tilde{\tau})^{K}(\tilde{\tau})^{-K}}^{\text {per }}(\xi)$ of (1.6) of $\tau \tau^{-1}$-type. This implies that there is neither periodicity nor symmetry in this pattern on $(0, L)=\left(0, \frac{T}{2}\right)$, although it will definitely look as if the 'block' $A_{1} B_{1} B_{1} A_{1} A_{1} B_{1}$ is repeated $K$ times in the output of a numerical simulation of the PDE. Moreover, the maxima of the $U$ and $V$ pulses cannot coincide, although that's again certainly not obvious from a simulation (see also [29]). A close inspection will show that there are asymptotically small differences between each of these $K$ 'blocks', and that the maxima of the $U$ and $V$ pulses have slightly different $x$-coordinates. This latter observation is especially clear, since coinciding maxima correspond to an intersection of the associated solution of (1.6) with $\{p=q=0\}$ in phase space. This would imply, by the symmetry (1.7), that the pattern should be symmetric around this point, which cannot be the case.

This perhaps subtle distinction can be expected to have a significant influence on the stability of the patterns on bounded intervals, since the associated linearized stability problem will, in general, have asymptotically small eigenvalues [29], see also Remark 5.1. This issue will be discussed in more detail in [28].

REMARK 5.1. In [29] bounded domain patterns without pulses at the boundary of the type $\tilde{\tau}=\sigma_{1} \sigma_{2} \ldots \sigma_{\tilde{J}}$, with $\sigma_{j} \in\left\{A_{1}, B_{1}\right\}$, have been analyzed, by a combination of (formal) asymptotic methods and numerical computations, as solutions of a simplified version of the generalized Gierer-Meinhardt equation (1.1). This simplified version of (1.1) has no explicit $U_{t}$ term, as a consequence the stability analysis simplifies considerably (for instance, the spectrum associated to the stability of these patterns is real; this is not the case in the 'full' equation [5]). In [28], the stability of the periodic patterns $\left(U_{\hat{\tau}}^{\text {per }}(x), V_{\hat{\tau}}^{\text {per }}(x)\right)$, with $\hat{\tau}=\tau \tau^{-1}, \tau \sigma \tau^{-1}$ or $\sigma \tau \tilde{\sigma} \tau^{-1}$, will be analyzed as solution of (1.1) on $\mathbf{R}$.

Acknowledgments. The authors thank Dick Hall for useful conversations about periodic symbol sequences. Partial support for this work was received from the Dutch Organization for Scientific Research (NWO), project 613-06-568, and National Science Foundation Grant DMS-0072596. 


\section{REFERENCES}

[1] J.G. Blom And P.A. ZEgeling, Algorithm 731: A moving-grid interface for systems of onedimensional time-dependent partial differential equations, ACM Transactions in Mathematical Software, 20 (1994), pp. 194-214.

[2] R. Camassa, G. KovaČIČ AND S.K. Tin, A Melnikov method for homoclinic orbits with many pulses, Arch. Rat. Mech. Analysis, 143 (1998), pp. 105-193.

[3] A. Doelman, Breaking the hidden symmetry in the Ginzburg-Landau equation, Physica D, 97 (1996), pp. 398-428.

[4] A. Doelman, R.A. Gardner AND T.J. KAPer, Stability analysis of singular patterns in the 1-D Gray-Scott model: A matched asymptotics approach, Physica D, 122 (1998), pp. 1-36.

[5] A. Doelman, R.A. Gardner AND T.J. KaPer, Large stable pulse solutions in reactiondiffusion equations, Indiana Univ. Math. J., 50 (1) (2001), pp. 443-507.

[6] A. Doelman and P. Holmes, Homoclinic explosions and implosions, Phil. Trans. R. Soc. Lond. A, 354 (1996), pp. 845-893.

[7] A. Doelman, T.J. Kaper and P. Zegeling, Pattern formation in the one-dimensional GrayScott model, Nonlinearity, 10 (1997), pp. 523-563.

[8] A. Doelman AND V. RotTschäFER, Singularly perturbed and nonlocal modulation equations for systems with interacting instability mechanisms, J. Nonlinear Sci., 7 (4) (1997), pp. 371-409.

[9] A. Doelman and H. VAn Der Ploeg, Homoclinic stripe patterns, to appear in SIAM J. Appl. Dyn. Sysú. (2001).

[10] N. Fenichel, Persistence and smoothness of invariant manifolds for flows, Indiana Univ. Math. J., 21 (1971), pp. 193-226.

[11] N. FENICHEL, Geometrical singular perturbation theory for ordinary differential equations, J. Diff. Eq., 31 (1979), pp. 53-98.

[12] R.A. GARDNER, Spectral analysis of long wavelength periodic waves and applications, J. reine angew. Math., 491 (1997), pp. 149-181.

[13] A. Gierer and H. Meinhardt, A theory of biological pattern formation, Kybernetik, 12 (1972), pp. 30-39.

[14] G. HEK, Fronts and pulses in a class of reaction-diffusion equations: a geometric singular perturbations approach, Nonlinearity, 14 (2001), pp. 35-72.

[15] G. Hek, A. Doelman and P. Holmes, Homoclinic saddle-node bifurcations and subshifts in a three-dimensional flow, Arch. Rat. Mech. An., 145 (1998), pp. 291-329.

[16] D. IRON AND M.J. WARD, A metastable spike solution for a non-local reaction-diffusion model, SIAM J. Appl. Math., 60(3) (2000), pp. 778-802.

[17] D. IRON, M.J. WARD AND J. WEI, The stability of spike solutions of the one-dimensional Gierer-Meinhardt model, Phys. D, 150 (2001), pp. 25-62.

[18] C.K.R.T. Jones, Geometric singular perturbation theory, in 'Dynamical systems, Montecatibi Terme, 1994' (R. Johnson ed.), Lecture Notes in Mathematics, 1609 (1995) (Springer), pp. 44-118.

[19] C.K.R.T. Jones, T.J. KAPER AND N. KoPELL, Tracking invariant manifolds up to exponentially small errors, SIAM J. Math. Anal., 27(2) (1996), pp. 558-577.

[20] T.J. KAPER, An introduction to geometric methods and dynamical systems theory for singular perturbation problems, in 'Analyzing Multiscale Phenomena Using Singular Perturbation Methods' (R.E. O'Malley, J. Cronin eds.), Proc. of Symposia in Appl. Math., 56 (1999) (American Mathematical Society), pp. 85-132.

[21] T.J. Kaper And G. KovaČIČ, Multi-bump orbits homoclinic to resonance bands, Trans. Amer. Math. Soc., 348 (10) (1996), pp. 3835-3887.

[22] D.S. Morgan, A. Doelman And T.J. KaPer, Stationary periodic orbits in the 1-D Gray-Scott model, Meth. Appl. Anal., 7(1) (2000), pp. 105-150.

[23] W.-M. NI, Diffusion, cross-diffusion, and their spike-layer steady states, Notices AMS, 45(1) (1998), pp. 9-18.

[24] W.-M. Ni AND I. TAKagi, Point condensation generated by a reaction-diffusion system in axially symmetric domains, Japan J. Industr. Appl. Math., 12 (1995), pp. 327-365.

[25] C. RoBInSon, Sustained resonance for a nonlinear system with slowly-varying coefficients, SIAM J. Math. Anal., 14 (1983), pp. 847-860.

[26] C. Soto-Treviño, A geometric method for periodic orbits in singularly perturbed systems, in 'Multiple-Time-Scale Dynamical Systems' (C.K.R.T. Jones and A. Khibnik, eds.), IMA Volumes in Mathematics and its Applications, 122 (2000) (Springer, NY), pp. 141-202.

[27] C. Soto-Treviño And T.J. KaPer, Periodic orbits in singularly-perturbed systems, in 'Nonlinear Dynamics and Pattern Formation in the Natural Environment' (A. Doelman, A. van 
Harten eds.), Pitman Res. Notes Math., 335 (1995) (Longman, Harlow), pp. 295-314.

[28] H. van Der Ploeg AND A. Doelman, in preparation.

[29] M.J. WARD AND J. WEI, Asymmetric spike patterns for the one-dimensional Gierer-Meinhardt model: equilibria and stability, submitted.

[30] J. WEI, On single interior spike solutions of the Gierer-Meinhardt system: uniqueness and spectrum estimates', European J. Appl. Math., 10(4) (1999), pp. 353-378. 